OPEN ACCESS

Edited by:

Dayun Tao,

Yunnan Academy of Agricultural

Sciences, China

Reviewed by:

Cengiz Toker,

Akdeniz University, Turkey

Devendra Ram Malaviya,

Indian Grassland and Fodder

Research Institute (ICAR), India

*Correspondence:

Naveen Singh

ns1.genet@gmail.com

Specialty section:

This article was submitted to

Plant Breeding,

a section of the journal

Frontiers in Plant Science

Received: 27 August 2021 Accepted: 13 October 2021

Published: 25 November 2021

Citation:

Limbalkar OM, Singh R, Kumar $P$, Nanjundan J, Parihar CM, Vasisth $P$,

Yadava DK, Chinnusamy $V$ and

Singh N (2021) Deployment

of Brassica carinata A. Braun Derived

Brassica juncea (L.) Czern. Lines

for Improving Heterosis and Water Use Efficiency Under Water Deficit

Stress Conditions.

Front. Plant Sci. 12:765645. doi: 10.3389/fpls.2021.765645

\section{Deployment of Brassica carinata A. Braun Derived Brassica juncea (L.) Czern. Lines for Improving Heterosis and Water Use Efficiency Under Water Deficit Stress Conditions}

\author{
Omkar Maharudra Limbalkar', Rajendra Singh ${ }^{1}$, Parvesh Kumar', Joghee Nanjundan', \\ Chiter Mal Parihar ${ }^{3}$, Prashant Vasisth ${ }^{1}$, Devendra Kumar Yadava ${ }^{4}$, \\ Viswanathan Chinnusamy ${ }^{5}$ and Naveen Singh ${ }^{1 *}$
}

\begin{abstract}
'Division of Genetics, Indian Council of Agricultural Research-Indian Agricultural Research Institute, New Delhi, India, ${ }^{2}$ Indian Council of Agricultural Research-Indian Agricultural Research Institute Regional Station, Wellington, India, ${ }^{3}$ Division of Agronomy, Indian Council of Agricultural Research-Indian Agricultural Research Institute, New Delhi, India, ${ }^{4}$ ADG (Seeds), Indian Council of Agricultural Research, New Delhi, India, ${ }^{5}$ Division of Plant Physiology, Indian Council of Agricultural

Research-Indian Agricultural Research Institute, New Delhi, India
\end{abstract}

Among Brassica species, Ethiopian mustard (Brassica carinata A. Braun) is known to tolerate most abiotic stresses, including drought. Drought caused by low and erratic rainfall in semi-arid regions consistently challenges rapeseed mustard productivity. Development of $B$. carinata-derived lines (CDLs) in Brassica juncea (L.) Czern. nuclear background, carrying genomic segments from B. carinata, are expected to tolerate moisture deficit stress conditions. The present study was, thus, aimed to establish the phenomenon "heterosis" for drought tolerance and water use efficiency by evaluating 105 hybrids developed from intermating 15 CDLs in half diallel fashion. Data on 17 seed yield and yield contributing traits were recorded under two different environments, viz., irrigated and rainfed conditions. Traits under study were found to be governed by both additive and non-additive types of gene action. Average degree of dominance was higher (>2) for yield and yield contributing traits, viz., secondary branches/plant, point to first siliqua on main shoot, total siliquae/plant, 1,000-seed weight, seed yield/plant, biological yield, harvest index, and seed yield/hectare under rainfed conditions, clearly indicating that higher productivity under drought conditions can be realised through the development of hybrids. Out of 15 , highly significant general combining ability (GCA) effects for seven CDLs were observed under rainfed condition. Furthermore, nine and six hybrids expressed highly significant specific combining ability (SCA) effects and $>50 \%$ heterobeltiosis for yield contributing traits under rainfed and irrigated conditions, respectively. Water use efficiency (WUE) of parental CDLs and hybrids varied from 2.05 to $2.57 \mathrm{~kg} \mathrm{~m}^{-3}$ under rainfed, while 1.10 to $1.28 \mathrm{~kg} \mathrm{~m}^{-3}$ under irrigated conditions. Hybrids expressed higher WUE than parental lines under both water regimes. Furthermore, selection indices such as drought tolerance index (DTI) and mean relative performance (MRP) were identified to be efficient in the selection of productive 
CDLs and hybrids under drought conditions. Nine hybrids, identified as highly productive in the present study, can further be exploited for improving the yield of Indian mustard in drought-prone areas. Usefulness of interspecific hybridisation in the development of $B$. carinata-derived $B$. juncea lines for improving heterosis and WUE is, thus, well demonstrated through the present study.

Keywords: Brassica juncea, interspecific hybridization, gene effects, heterosis, water use efficiency, drought tolerance

\section{INTRODUCTION}

Rapeseed-mustard group is the second most important oilseed crop in the world after soybean with a production of 69.08 million metric tons and contributed $11.89 \%$ to global oilseed production (580.51 million metric tons) in 2019-2020 (Anonymous, 2021). India is the third-largest producer of rapeseed-mustard group of crops followed by Canada and China with a total production of 7.4 million metric tons from a $7.1 \mathrm{mha}$ area (Anonymous, 2021). Indian mustard, Brassica juncea (L.) Czern. (AABB; $2 \mathrm{n}=36$ ), is an amphidiploid species that originated from a spontaneous interspecific hybridisation of Brassica rapa $\mathrm{L} .(\mathrm{AA}, 2 \mathrm{n}=20)$ and B. nigra $\mathrm{L}$. $(\mathrm{BB}, 2 \mathrm{n}=16)$. It is a widely cultivated species among the rapeseed-mustard group of crops accounting for more than $90 \%$ of its total acreage in India. Being a pivotal crop of the Indian oilseed sector, extensive efforts have been made to improve its seed and oil yields to achieve national self-sufficiency in edible oils. Despite that, a huge amount of edible oil is being imported to meet the requirements for domestic consumption. The Solvent Extractors' Association of India reported that the import of edible oil rises to 23 million metric tons, costing more than 11 thousand million dollars in 2020 in the country, which accounts for more than $60 \%$ of the total edible oil demand.

To achieve self-sufficiency in edible oils, there is an urgent need to improve the productivity of Indian mustard. However, narrow genetic base of working gene pool is a major impediment in the improvement of this species (Chauhan et al., 2011). Most of the released Indian varieties are either derived directly from the cultivar Varuna or its derivatives (Singh and Chauhan, 2010; Chauhan et al., 2011). The yield potential of these varieties is about $4 \mathrm{t} / \mathrm{ha}$; however, the national average yield is hovering around $1.5 \mathrm{t} / \mathrm{ha}$. The stochastic production patterns are largely attributed to its susceptibility to different biotic and abiotic factors (Sharma et al., 2018). Indian mustard group of crops is mainly cultivated by marginal and small-farmers, wherein about $25 \%$ of the rapeseed-mustard area is rainfed, causing critical yield losses particularly in the drought-prone areas of eastern and western parts of the country (Sharma et al., 2018). Most of the cultivars of Indian mustard are susceptible to drought and encounter severe yield losses up to $94 \%$ (Sharma and Kumar, 1989). Although few varieties of Indian mustard have been released for commercial cultivation in rainfed areas of the country (Chauhan et al., 2011), these varieties failed to occupy sufficiently large area in the drought prone regions. This indicates that target environments still demand a better level of tolerance in future varieties. This also calls for the urgent development of high-yielding water use efficient genotypes and hybrids which can withstand drought conditions to minimise the yield losses, improve productivity and stabilise mustard production.

Relatives of B. juncea [Sinapis alba (Warwick, 1993), Arabidopsis thaliana (Kasuga et al., 1999); B. rapa (Seo et al., 2010), B. napus (Dalal et al., 2009; Fletcher et al., 2015), B. carinata (Malik, 1990)] harbour many useful traits, such as tolerance to drought, salinity, and cold. These could be used to incorporate abiotic stress tolerance in present-day cultivars (Warwick, 1993). Furthermore, wide hybridisation has been considered a novel method for generating selectable genetic variability in cultivated species (Prakash, 1973; Inomata, 1997).

Brassica carinata (BBCC; $2 \mathrm{n}=34$ ), an important oilseed crop of Ethiopian origin, is being cultivated in northeast Africa, parts of Canada, France, Spain, Australia, China, and India. Although this species has tall plant stature, poor seed characteristics, and takes longer to mature (Supplementary Table 1), when compared to $B$. juncea, it possesses many agronomically desirable traits including resistance or tolerance to most of the abiotic and biotic stresses like drought, heat, aphid, Sclerotinia rot, white rust, Alternaria black spot, blackleg, and powdery mildew (Raman et al., 2017; Thakur et al., 2019), which are otherwise eliminated from the predominantly cultivated Brassica species during domestication. Therefore, efforts toward interspecific hybridisation were made in the past to transfer desirable traits from B. carinata to cultivated Brassica species viz., B. rapa (Jiang et al., 2007; Choudhary et al., 2008), B. napus (Navabi et al., 2011; Sheikh et al., 2014), and B. juncea (Getinet et al., 1994; Sheikh et al., 2014; Singh K. H. et al., 2015). B. carinata is known to be the most drought tolerant among cultivated Brassica species as it develops sufficient biomass and seed yield even under the water deficit stress conditions. However, rainfall or irrigation leads to higher biomass accumulation, lodging of crop and, thus, reduction in seed size, oil content, and other yield related traits in this species (Thakur et al., 2019).

Keeping this in view, efforts were made at the Indian Council of Agricultural Research-Indian Agricultural Research Institute (ICAR-IARI), New Delhi to enrich the genome of B. juncea with $B$. carinata, through the development of $B$. carinataderived B. juncea lines (CDLs), for improving their ability to withstand drought conditions. Though inter-subgenomic heterosis were reported in hybrids of $B$. rapa and B. carinataderived introgression lines with $B$. juncea accessions (Wei et al., 2016), the present study aimed to use B. carinata-derived $B$. juncea lines for improving heterosis and water use efficiency (WUE) under water deficit stress conditions. To the best of our knowledge, this is the first case where diverse CDLs were developed and deployed for improving drought tolerance. 
Hybrid breeding involving diverse parental lines is considered one of the most viable options for breaking the yield barrier (Singh N. et al., 2015). Involvement of alien introgression lines in hybrid development enables stretching of genetic diversity among parental lines required for the expression of higher heterosis, complementation of favorable novel alleles from both the introgressed parents in the $\mathrm{F}_{1}$ generation, and, thus, improved ability of hybrids to withstand stress. Information on gene action, their relative contribution to the genetic variance, and estimation of general and specific combining ability effects (GCA and SCA) under water deficit stress conditions are pre-requisite for the identification of best parental combinations enabling the development of high yielding drought tolerant mustard hybrids (Panhwar et al., 2008; Khan et al., 2009). Several selection indices have also been suggested for the identification of water use efficient genotypes and cross combinations in crop plants, including mustard (Fischer and Maurer, 1978; Rosielle and Hamblin, 1981; Clarke et al., 1984; Fernandez, 1992; Huang, 2000). Despite this, this information was not much helpful in mitigating the ill-effect imposed by drought conditions in Indian mustard, primarily due to inherent genetic ceiling in this species.

Brassica juncea CDLs possessing useful genomic segment(s) from B. carinata, developed at the Indian Council of Agricultural Research - Indian Agricultural Research Institute (ICAR-IARI), New Delhi, shall be useful in the identification of high yielding drought tolerant parental lines and hybrids. Furthermore, information generated on gene action and degree of dominance for yield contributing traits and WUE under moisture deficit stress condition in $B$. carinata derived lines and hybrids shall help in devising future breeding strategies. The present study was, therefore, conducted to (i) determine the type of gene action, combining ability of parental lines and heterobeltiosis in the hybrids developed by using CDLs, (ii) assess the WUE of CDLs and their hybrids, and (iii) identify water use efficient genotypes and cross combinations.

\section{MATERIALS AND METHODS}

\section{Selection of Brassica carinata Derived Brassica juncea Lines}

A set of $F_{2}$ populations was generated from crossing B. juncea genotypes (DRMRIJ 31, Pusa Mustard 30 and Pusa Agrani) with B. carinata accessions (BC-4, BC-5, and BC-12). Within each of these $\mathrm{F}_{2}$ populations, plants were selected on the basis of phenotype and were inter-mated in pairs - a method commonly called biparental mating. Phenotypic selection in the subsequent filial generations led to the development of $B$. carinata-derived $B$. juncea lines (CDLs). These cytologically stable homozygous CDLs possess 18 bivalents $(2 \mathrm{n}=36)$, indicating its similarity to $B$. juncea (Supplementary Figures 1, 2). These CDLs, in $\mathrm{F}_{6}$ generation, were evaluated in two rows of 4-m length plots under both irrigated and rainfed field condition in the Division of Genetics, ICAR- IARI, New Delhi during 2018-2019 rabi season. To select CDLs differing in metric traits and response to moisture deficit stress, row-row and plant-plant spacing were kept at 30 and $15 \mathrm{~cm}$, respectively (data not presented). One row was left blank before and after every plot such that plotplot and row-row in a plot were separated by a spacing of 60 and $30 \mathrm{~cm}$, respectively. The experiment was raised in augmented design both under irrigated and rainfed conditions. No irrigation was applied in the rainfed plot, while two irrigations, each of $50 \mathrm{~mm}$ depth, were applied in irrigated plots at 45 and 90 days after sowing (DAS). All recommended agronomic practices were adopted for raising the crop. Based on drought tolerance indices, 15 CDLs differing for various morpho-physiological traits and phenotypical and cytological resemblance to B. juncea (Supplementary Table 1 and Supplementary Figures 1, 2) were selected for generating hybrids.

\section{Generation of Crosses}

During 2019, 15 diverse CDLs differing for morphophysiological traits were intermated in half diallel design (excluding reciprocals) to generate seeds of $105 \mathrm{~F}_{1}$ hybrids in ICAR-IARI, Regional Station, Wellington, Tamil Nadu, India in the off-season.

\section{Evaluation of Hybrids and Their Parental Lines}

During rabi 2019-2020 season, the set of $105 \mathrm{~F}_{1}$ hybrids along with 15 parental CDLs was raised under both rainfed and irrigated conditions in the research field of Division of Genetics, ICAR-IARI, New Delhi. Two recommended irrigations of $50 \mathrm{~mm}$ depth each were applied at 45 and 90 DAS to the irrigated plots, while no irrigation was given to rainfed plots. Hybrids along with parents were raised in a randomised complete block design (RCBD) with three replications. Each plot consisted of a paired row of $4 \mathrm{~m}$ in length. Row to row and plant to plant distance were kept 30 and $15 \mathrm{~cm}$, respectively. One row of $30 \mathrm{~cm}$ was left blank before and after every pair-row plot such that plot-plot and row-row in a plot were distanced at 60 and $30 \mathrm{~cm}$, respectively. Recommended agronomical practices and plant protection measures were adopted for raising the crop.

Observations were recorded on 17 quantitative traits, viz., plant height $(\mathrm{cm})$, point to first branch $(\mathrm{cm})$, number of primary branches, number of secondary branches, main shoot length $(\mathrm{cm})$, point to first siliqua on main shoot $(\mathrm{cm})$, number of siliquae on main shoot, total siliquae per plant, siliqua length $(\mathrm{cm})$, seeds per siliqua, days to $50 \%$ flowering, 1,000-seed weight $(\mathrm{g})$, seed yield per plant (g), biological yield per plant (g), harvest index, seed yield ( $\mathrm{kg} / \mathrm{ha})$, and oil content. The data were recorded on five randomly selected competitive plants from all the plots in each replication, except for seed yield per plot $(\mathrm{g})$ and days to $50 \%$ flowering where observations were recorded on plot basis. Biological yield was recorded at physiological maturity after drying the harvested plants. Data on plot yield (g) were used to calculate seed yield (kg/ha).

\section{Assessment of Drought Tolerance}

Data were recorded on 17 metric traits under both irrigated and rainfed conditions. Mean values of these traits were used to analyse drought susceptibility index (DSI; Fischer and Maurer, 1978), drought tolerance index (DTI; Fernandez, 1992), tolerance 
index (TOL; Rosielle and Hamblin, 1981), and mean relative performance (MRP) for the CDLs taken in this study (Raman et al., 2012).

Water-use efficiency (WUE) for parents and hybrids were calculated from the below given equation:

WUE $\left[\mathrm{kg} \mathrm{ha}^{-1} \mathrm{~mm}^{-1}\right]=$ Seed Yield $\left(\mathrm{kgha}^{-1}\right) /$ Water received from irrigation and rainfall $(\mathrm{mm})$

$$
\text { and WUE }\left[\mathrm{kg} \mathrm{m}^{-3}\right]=0.1 \times \text { WUE }\left[\mathrm{kg} \mathrm{ha}^{-1} \mathrm{~mm}^{-1}\right] \text {. }
$$

The effective rainfall (between October 2019 and February 2020) was calculated by using Cropwat Version 8.0 by USDA SCS method. Cropwat is a computer-based decision support tool developed by the Food and Agriculture Organization (FAO) of the United Nations to estimate effective rainfall based on climate and soil data. The maximum and minimum temperatures and rainfall $(\mathrm{mm})$ recorded during 2019-2020 crop seasons from ICAR-IARI meteorological observatory, located within $500 \mathrm{~m}$ distance from the experimental site, is presented in Supplementary Figure 3.

\section{Statistical and Genetic Analyses}

The mean values obtained from 17 different quantitative traits were subjected to analysis of mean and variance. Data obtained from $105 \mathrm{~F}_{1}$ and 15 parental CDLs were subjected to Griffing's method II with a fixed model (model I) to determine the combining abilities (Griffing, 1956). The mathematical model for ijkth observation is as follows:

$$
Y i j=\mu+g_{i}+g_{j}+s_{i j}+b_{k}+e_{i j k}
$$

where $Y_{i j}$ is the mean of $i \times j^{\text {th }}$ genotype over $k ; \mu$ is general population mean; $g_{i}$ and $g_{j}$ is the general combining ability effects of $i^{\text {th }}$ and $j^{\text {th }}$ parent, respectively; $s_{i j}$ is the specific combining ability effects of cross involving $i^{\text {th }}$ and $j^{\text {th }}$ parent, respectively; and $b_{k}$ is the replication effect; and $\mathrm{e}_{i j k}$ is the residual error.

Heterobeltiosis expressed in the hybrids was calculated by using mean values of respective $F_{1}$ and its better parent (BP) following standard formula (Coffman, 1933). The narrowsense heritability $\left(h^{2}\right)$ was calculated by using the method suggested by Mather and Jinks (1982). Analysis of variance and estimation of degree of dominance, heritability, and combining ability were done following the method suggested by Griffing (1956) using Windostat Version 9.1. The degree of dominance was estimated from variance due to dominance $\left(\sigma^{2} D\right)$ and variance due to additive $\left(\sigma^{2} A\right)$ gene action by using the formula $\left[\left\{\left(2 \times \sigma_{D}^{2}\right) / \sigma_{A}^{2}\right\}^{1 / 2}\right]$ given by Comstock and Robinson (1948).

\section{RESULTS}

Sufficient phenotypic variation was observed for the seed yield and its contributing traits under both rainfed and irrigated conditions. Most of the traits were normally distributed except days to $50 \%$ flowering (Supplementary Figure 4 ). There were significant differences $(p<0.001)$ among the parents and hybrids for all the recorded 17 traits, viz., plant height, point to the first branch, number of primary branches per plant, number of secondary branches per plant, main shoot length, point to the first siliqua on the main shoot, number of siliquae on the main shoot, total number of siliquae per plant, days to $50 \%$ flowering, 1,000-seed weight (g), seed yield per plant (g), biological yield per plant (g), harvest index, seed yield ( $\mathrm{kg} / \mathrm{ha})$, and oil content (\%) under irrigated (Table 1) and rainfed (Table 2) conditions. The mean squares of parents vs. hybrids under irrigated condition were found to be significant for all traits except point to first siliqua on the main shoot while under the rainfed condition, it was also significant for most of the traits except point to the first siliqua on the main shoot, seeds per siliqua, 1,000-seed weight, and oil content. Results also exhibited a significant mean sum of squares due to hybrids for all the traits under both rainfed and irrigated conditions (Tables 1, 2).

The variance due to specific combining ability $\left(\sigma^{2} s c a\right)$ were higher than general combining ability variance $\left(\sigma^{2}\right.$ gca) for traits, viz., point to the first branch, number of primary branches, number of secondary branches, main shoot length, point to first siliqua on the main shoot, number of siliquae on the main shoot, total siliquae per plant, days to $50 \%$ flowering, 1,000-seed weight $(\mathrm{g})$, seed yield per plant $(\mathrm{g})$, biological yield per plant $(\mathrm{g})$, harvest index, seed yield $(\mathrm{kg} / \mathrm{ha})$, and oil content $(\%)$ and, thus, the average degree of dominance for these traits were observed to be more than one (Table 3). For siliqua length and seeds per siliqua under both irrigated and rainfed conditions, the average degree of dominance was less than unity. For plant height, $\sigma^{2} \mathrm{sca}>\sigma^{2}$ gca and the degree of dominance was found to be more than one under irrigated, whereas $\sigma^{2}$ gca $>\sigma^{2}$ sca and degree of dominance was less than unity under rainfed condition.

Narrow-sense heritability $\left(\mathrm{h}^{2}\right)$ for different traits varied from 0.09 to 0.69 and 0.07 to 0.75 under irrigated and rainfed conditions, respectively (Table 3 ). The estimate of the degree of dominance varied from 0.74 to 4.40 and from 0.54 to 5.13 under irrigated and rainfed conditions respectively across the traits (Table 3). Low to moderate $h^{2}$ were observed for all the traits under irrigated and rainfed conditions, with few exceptions. High average degree of dominance along with low $h^{2}$ were observed for point to first siliqua on main shoot $(\mathrm{cm})$, total number of siliquae per plant, seed yield per plant (g), biological yield per plant (g), and seed yield $(\mathrm{kg} / \mathrm{ha})$.

\section{General Combining Ability}

The general combining ability analysis revealed that each CDL possessed significant GCA effects for one or more traits under both irrigated or rainfed conditions (Table 4). CDL89 and CDL141 were found to be a good general combiner for point to the first siliqua on the main shoot, harvest index, seed yield per plant, seed yield $(\mathrm{kg} / \mathrm{ha})$, and oil content under rainfed conditions. CDL161, CDL112, CDL186, CDL101, and CDL25 were also found to possess high GCA for seed yield (kg/ha) and some of its contributing traits under water deficit stress conditions (Table 4).

Under irrigated condition, CDL161 and CDL106 expressed good general combining ability for point to the first branch, secondary branches/plant, main shoot length, total siliquae/plant, days to $50 \%$ flowering, 1,000 -seed weight, seed yield/plant, biological yield/plant, and seed yield (kg/ha) (Table 4). CDL112, CDL186, CDL101, CDL25, and CDL89 also possessed higher 
TABLE 1 | Analysis of variance for parents and hybrids generated in half diallel design for studying different yield contributing traits under irrigated conditions.

\begin{tabular}{|c|c|c|c|c|c|c|c|c|}
\hline \multirow[b]{2}{*}{ Traits } & \multicolumn{5}{|c|}{ Sources of variations } & \multirow[b]{2}{*}{ Error } & \multirow[b]{2}{*}{ Mean } & \multirow[b]{2}{*}{ CV (\%) } \\
\hline & Replications & Female:male & Parents & Hybrids & Parents Vs. Hybrids & & & \\
\hline \multirow[t]{2}{*}{$\mathrm{PH}$} & 53.50 & $764.78^{\star \star}$ & $1047.12^{\star \star}$ & $660.99^{\star \star}$ & $7605.9^{\star \star}$ & 140.32 & 230.07 & 5.15 \\
\hline & (0.38) & $(5.45)$ & $(7.46)$ & $(4.71)$ & $(54.2)$ & & & \\
\hline PFB & 12.47 & $717.21^{\star \star}$ & $685.04^{\star \star}$ & $726.95^{\star \star}$ & $155.17^{\star \star}$ & 5.72 & 32.34 & 7.39 \\
\hline $\mathrm{PB} / \mathrm{pt}$ & (0.69) & (3.96) & (2.52) & $(4.01)$ & (18.56) & & & \\
\hline \multirow[t]{2}{*}{$\mathrm{SB} / \mathrm{pt}$} & 3.81 & $51.78^{\star \star}$ & $34.41^{\star \star}$ & $50.68^{\star \star}$ & $409.29^{\star \star}$ & 1.71 & 17.89 & 7.32 \\
\hline & $(2.23)$ & $(30.2)$ & (20.07) & (29.56) & (238.68) & & & \\
\hline \multirow[t]{2}{*}{ MSL } & 4.41 & $185.65^{\star \star}$ & $104.47^{\star \star}$ & $190.97^{\star \star}$ & $768.96^{\star \star}$ & 37.81 & 79.73 & 7.71 \\
\hline & $(0.12)$ & $(4.91)$ & $(2.76)$ & $(5.05)$ & (20.33) & & & \\
\hline \multirow[t]{2}{*}{ TS/pt } & 271.49 & $79803.8^{* \star}$ & $29008.97^{\star \star}$ & $84878.04^{\star \star}$ & $263209.97^{\star \star}$ & 1443.53 & 529.02 & 7.18 \\
\hline & $(0.19)$ & $(55.28)$ & (20.09) & $(58.79)$ & (182.33) & & & \\
\hline \multirow[t]{2}{*}{ Siliqua length } & 0.127 & $0.517^{\star \star}$ & $0.697^{\star \star}$ & $0.49^{\star \star}$ & $0.781^{\star \star}$ & 0.062 & 4.15 & 5.99 \\
\hline & (2.06) & $(8.37)$ & $(11.27)$ & (7.93) & (12.64) & & & \\
\hline \multirow[t]{2}{*}{ Seeds/Siliqua } & 0.55 & $7.5^{\star \star}$ & $8.33^{\star \star}$ & $7.17^{\star \star}$ & $30.63^{\star \star}$ & 1.55 & 15.89 & 7.85 \\
\hline & $(0.35)$ & (4.83) & (5.35) & $(4.61)$ & (19.69) & & & \\
\hline \multirow[t]{2}{*}{ D50\%F } & 3.13 & $107.05^{\star \star}$ & $116.8^{\star \star}$ & $98.61^{\star \star}$ & $848.42^{\star \star}$ & 12.69 & 56.73 & 6.28 \\
\hline & $(0.25)$ & $(8.43)$ & (9.19) & $(7.76)$ & (66.81) & & & \\
\hline \multirow[t]{2}{*}{ TSW } & 0.17 & $0.87^{\star \star}$ & $0.802^{\star \star}$ & $0.851^{\star \star}$ & $3.46^{\star \star}$ & 0.079 & 3.96 & 7.1 \\
\hline & (2.13) & (10.94) & (10.12) & (10.73) & (43.68) & & & \\
\hline SY/pt & 2.51 & $302.77^{\star \star}$ & $50.39^{\star \star}$ & $303.21^{\star \star}$ & $3790.15^{\star \star}$ & 12.63 & 28.94 & 12.28 \\
\hline \multirow[t]{2}{*}{ Oil content } & 7.06 & $17.77^{\star \star}$ & $27.27^{\star \star}$ & $15.26^{\star \star}$ & $146^{\star \star}$ & 2.4 & 33.32 & 4.66 \\
\hline & (2.93) & (7.37) & (11.31) & (6.33) & $(60.57)$ & & & \\
\hline
\end{tabular}

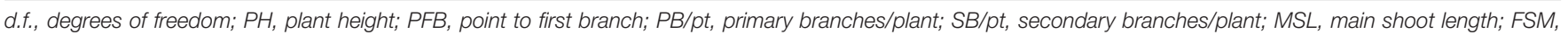

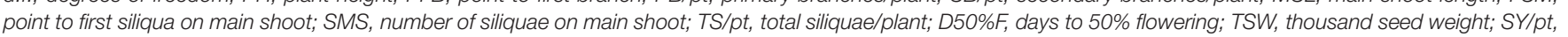
seed yield/plant; BY/pt, biological yield/plant; HI, harvest index; SY/ha, seed yield kg per hectare.

** Significant at $p=0.01$ and *significant at $p=0.05$.

GCA for seed yield (kg/ha) along with some of its contributing traits under water sufficient conditions (Table 4).

\section{Specific Combining Ability and Heterobeltiosis}

Under the rainfed condition, a total of 36 hybrids exhibited significant SCA effects and high heterobeltiosis (>15\%) for seed yield ( $\mathrm{kg} / \mathrm{ha})$. Highest heterobeltiosis (99.55\%) was observed for the cross CDL106 $\times$ CDL104 under limited water conditions. Out of 36, nine hybrids, viz., CDL102 × CDL89, CDL102 × CDL106, CDL112 × CDL89, CDL186 × CDL121, CDL182 × CDL121, CDL $121 \times$ CDL 103, CDL25 $\times$ CDL104, CDL89 $\times$ CDL104, and CDL104 $\times$ CDL106 exhibited highly significant SCA effects and more than 50\% heterobeltiosis (Table 5). Similarly, under irrigated condition, 30 hybrids expressed significant SCA effects and heterobeltiosis > 15\%. Highest heterobeltiosis $(82.34 \%)$ for seed yield $(\mathrm{kg} / \mathrm{ha})$ was observed for the hybrid derived from parents CDL141 and CDL161 under favourable environment. Six hybrids, viz., CDL161 × CDL186, CDL161 × CDL141, CDL141 × CDL182, CDL182 × CDL103, CDL182 × CDL106, and CDL104 $\times$ CDL106 recorded highly significant SCA effects and $>50 \%$ heterobeltiosis for seed yield $(\mathrm{kg} / \mathrm{ha})$ under irrigated condition. It is also important to note that hybrid derived from cross CDL104 $\times$ CDL106 observed highly significant SCA effects and $>50 \%$ heterobeltiosis under both rainfed and irrigated conditions (Table 5). 
TABLE 2 | Analysis of variance for parents and hybrids generated in half diallel design for studying different yield contributing traits under rainfed conditions.

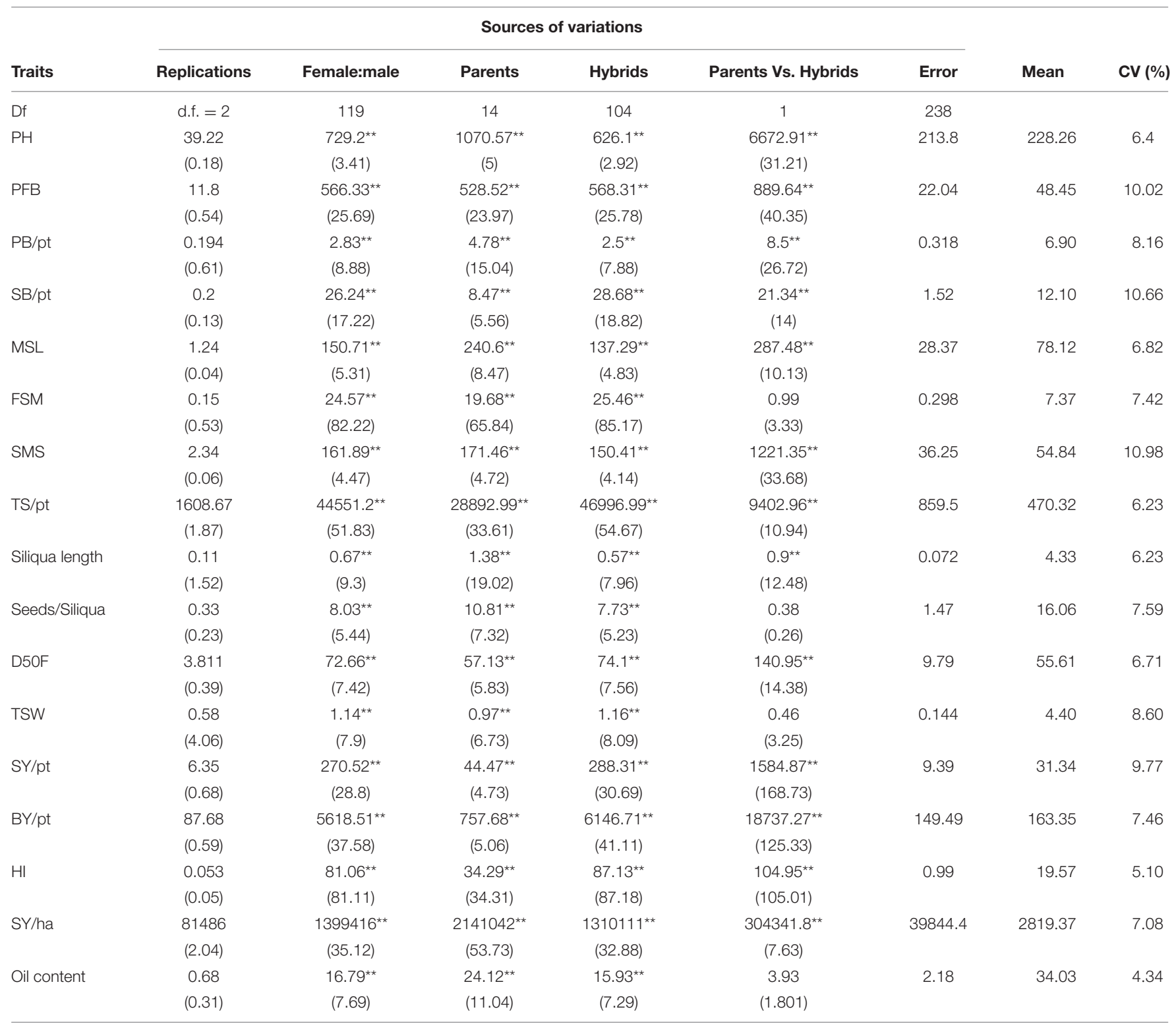

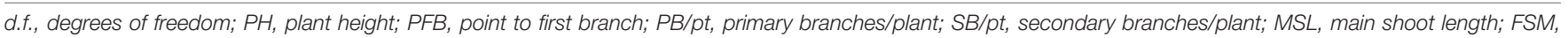

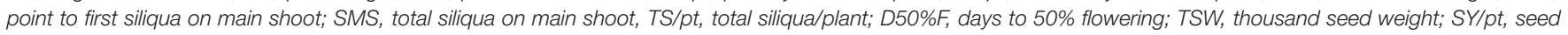
yield/plant; BY/pt, biological yield/plant; HI, harvest index; SY/ha, seed yield kg per hectare.

**Significant at $p=0.01$ and *significant at $p=0.05$.

High heterobeltiosis (>15\%) and higher SCA effects for seed yield were reported for hybrids derived from different combinations of parental CDLs, irrespective of their GCA effect levels (Table 6). Furthermore, 14 hybrids expressed significant SCA effects and high heterobeltiosis (>15\%) under both rainfed and irrigated conditions (Table 6).

\section{Mean Performance}

The water requirement of rapeseed mustard is about 190$400 \mathrm{~mm}$ (Shekhawat et al., 2012; Sharma et al., 2018) and it is sensitive to water deficit stress at critical stages like pre-flowering and siliquae formation stages. In the presented experiment, the rainfed plots received $112.4 \mathrm{~mm}$ of water through rains up to physiological maturity stage, whereas two additional irrigations of $50 \mathrm{~mm}$ each were applied in the irrigated plots. Thus, the irrigated plots received a total of $212.4 \mathrm{~mm}$ of water throughout the crop developmental stages and met optimum water requirement. The data recorded on 17 morphophysiological traits under rainfed and irrigated conditions were averaged separately for 15 parents and 105 hybrids. The results suggested that parental CDLs exhibited a higher mean value under irrigated than in rainfed condition for traits like plant height $(\mathrm{cm})$, number of primary branches, number of secondary branches, main shoot length $(\mathrm{cm})$, point to first siliqua on the 
main shoot $(\mathrm{cm})$, total siliquae per plant, days to $50 \%$ flowering, and seed yield (kg/ha). Whereas, under the rainfed condition, the mean value of CDLs was higher for point to first branch $(\mathrm{cm})$, siliquae on the main shoot, siliqua length $(\mathrm{cm})$, seeds/siliqua, 1,000-seed weight (g), seed yield/plant ( $\mathrm{g})$, biological yield/plant (g), harvest index, and oil content traits (Table 7) than in irrigated condition. Under irrigated condition, the overall mean of the hybrids was higher for plant height, primary branches per plant, secondary branches per plant, main shoot length, point to first siliqua on main shoot, total siliquae per plant and days to $50 \%$ flowering than in rainfed condition. Whereas traits like point to first branch $(\mathrm{cm})$, number of siliquae on the main shoot, siliqua length $(\mathrm{cm})$, seeds per siliqua, 1,000-seed weight $(\mathrm{g})$, seed yield per plant (g), biological yield per plant (g), harvest index, seed yield (kg/ha), and oil content (\%) observed higher mean values for hybrids when evaluated under rainfed condition (Table 7). Overall performance of CDLs and hybrids was found better when evaluated under rainfed condition for important yield and yield attributing traits including plant height $(\mathrm{cm})$, total siliquae on main shoot (no.), siliqua length $(\mathrm{cm})$, seeds/siliqua, days to $50 \%$ flowering, 1,000-seed weight ( $\mathrm{g}$ ), seed yield/plant, biological yield/plant (g), harvest index, and oil content (\%) traits (Table 7).

\section{Tolerance Indices and Water Use Efficiency}

Drought tolerance indices, viz., drought susceptibility index (DSI), drought tolerance index (DTI), tolerance index (TOL), and mean relative performance (MRP) for seed yield ( $\mathrm{kg} / \mathrm{ha})$ were estimated. Correlation coefficients between seed yield under

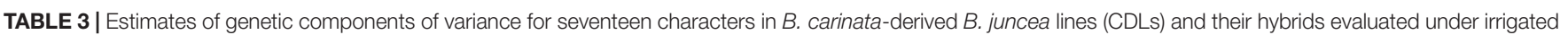
and rainfed conditions.

\begin{tabular}{|c|c|c|c|c|c|c|c|c|}
\hline Components of variance & & $\sigma^{2} g c a$ & $\sigma^{2} s c a$ & $\sigma_{g c a}^{2} / \sigma^{2} s c a$ & $\sigma^{2} A$ & $\sigma^{2} D$ & $\begin{array}{l}\text { Average degree of } \\
\text { dominance }\end{array}$ & $h^{2}$ \\
\hline \multirow[t]{2}{*}{ Plant height (cm) } & $\mathbb{R}$ & 69.75 & 77.81 & 0.90 & 139.50 & 77.81 & 1.06 & 0.52 \\
\hline & $\mathrm{RF}$ & 64.27 & 49.03 & 1.31 & 128.53 & 49.03 & 0.87 & 0.52 \\
\hline \multirow[t]{2}{*}{ Point to first branch (cm) } & $\mathbb{R}$ & 46.93 & 162.34 & 0.29 & 93.86 & 162.34 & 1.86 & 0.36 \\
\hline & $\mathrm{RF}$ & 36.22 & 123.53 & 0.29 & 72.44 & 123.53 & 1.85 & 0.36 \\
\hline \multirow[t]{2}{*}{ Primary branches/plant } & $\mathbb{R}$ & 0.19 & 0.59 & 0.33 & 0.39 & 0.59 & 1.75 & 0.30 \\
\hline & $\mathrm{RF}$ & 0.18 & 0.54 & 0.33 & 0.35 & 0.54 & 1.75 & 0.35 \\
\hline \multirow[t]{2}{*}{ Secondary branches/plant } & $\mathbb{R}$ & 0.88 & 16.93 & 0.05 & 1.75 & 16.93 & 4.40 & 0.09 \\
\hline & $\mathrm{RF}$ & 1.03 & 6.99 & 0.15 & 2.07 & 6.99 & 2.60 & 0.22 \\
\hline \multirow[t]{2}{*}{ Main shoot length (cm) } & $\mathbb{R}$ & 12.57 & 27.35 & 0.46 & 25.14 & 27.35 & 1.48 & 0.38 \\
\hline & $\mathrm{RF}$ & 7.22 & 29.84 & 0.24 & 14.44 & 29.84 & 2.03 & 0.27 \\
\hline \multirow[t]{2}{*}{ Point to first siliqua on main shoot $(\mathrm{cm})$} & $\mathbb{R}$ & 1.90 & 10.65 & 0.18 & 3.79 & 10.65 & 2.37 & 0.25 \\
\hline & $\mathrm{RF}$ & 0.57 & 7.86 & 0.07 & 1.14 & 7.86 & 3.71 & 0.13 \\
\hline \multirow[t]{2}{*}{ Total siliqua on main shoot } & $\mathbb{R}$ & 12.54 & 33.43 & 0.38 & 25.08 & 33.43 & 1.63 & 0.35 \\
\hline & $\mathrm{RF}$ & 7.67 & 30.06 & 0.26 & 15.34 & 30.06 & 1.98 & 0.27 \\
\hline \multirow[t]{2}{*}{ Total number of siliqua/plant } & $\mathbb{R}$ & 3917.17 & 20723.84 & 0.19 & 7834.34 & 20723.84 & 2.30 & 0.27 \\
\hline & $\mathrm{RF}$ & 1343.07 & 13461.46 & 0.10 & 2686.14 & 13461.46 & 3.17 & 0.16 \\
\hline \multirow[t]{2}{*}{ Siliqua length (cm) } & $\mathbb{R}$ & 0.06 & 0.03 & 1.85 & 0.12 & 0.03 & 0.74 & 0.69 \\
\hline & $\mathrm{RF}$ & 0.09 & 0.03 & 2.50 & 0.17 & 0.03 & 0.63 & 0.75 \\
\hline \multirow[t]{2}{*}{ Seeds/siliqua } & $\mathbb{R}$ & 0.73 & 0.60 & 1.22 & 1.46 & 0.60 & 0.91 & 0.57 \\
\hline & RF & 0.97 & 0.28 & 3.49 & 1.94 & 0.28 & 0.54 & 0.72 \\
\hline \multirow[t]{2}{*}{ Days to $50 \%$ flowering } & $\mathbb{R}$ & 8.98 & 15.29 & 0.59 & 17.96 & 15.29 & 1.30 & 0.48 \\
\hline & $\mathrm{RF}$ & 5.08 & 12.25 & 0.41 & 10.15 & 12.25 & 1.55 & 0.40 \\
\hline \multirow[t]{2}{*}{ 1,000-seed weight } & $\mathbb{R}$ & 0.05 & 0.19 & 0.26 & 0.10 & 0.19 & 1.95 & 0.32 \\
\hline & $\mathrm{RF}$ & 0.03 & 0.30 & 0.11 & 0.07 & 0.30 & 2.96 & 0.17 \\
\hline \multirow[t]{2}{*}{ Seed yield/plant } & $\mathbb{R}$ & 10.58 & 85.62 & 0.12 & 21.16 & 85.62 & 2.84 & 0.19 \\
\hline & $\mathrm{RF}$ & 7.27 & 82.16 & 0.09 & 14.54 & 82.16 & 3.36 & 0.15 \\
\hline \multirow[t]{2}{*}{ Biological yield/plant } & $\mathbb{R}$ & 1.88 & 1.92 & 0.98 & 3.76 & 1.92 & 1.01 & 0.16 \\
\hline & $\mathrm{RF}$ & 147.00 & 1732.25 & 0.08 & 294.00 & 1732.25 & 3.43 & 0.14 \\
\hline \multirow[t]{2}{*}{ Harvest index } & $\mathbb{R}$ & 2.09 & 7.29 & 0.29 & 4.18 & 7.29 & 1.87 & 0.35 \\
\hline & $\mathrm{RF}$ & 1.06 & 27.84 & 0.04 & 2.12 & 27.84 & 5.13 & 0.07 \\
\hline \multirow[t]{2}{*}{ Seed yield/hectare } & $\mathbb{R}$ & 18923.9 & 346993.1 & 0.05 & 37847.8 & 346993 & 4.28 & 0.10 \\
\hline & $\mathrm{RF}$ & 47004.54 & 407072.46 & 0.12 & 94009 & 407072 & 2.94 & 0.18 \\
\hline \multirow[t]{2}{*}{ Oil content } & $\mathbb{R}$ & 0.90 & 3.74 & 0.24 & 1.80 & 3.74 & 2.04 & 0.29 \\
\hline & RF & 1.58 & 1.94 & 0.81 & 3.16 & 1.94 & 1.11 & 0.54 \\
\hline
\end{tabular}

IR, irrigated; $R F$, rainfed; $h^{2}$, narrow sense heritability. 


\begin{tabular}{|c|c|c|c|c|c|c|c|c|c|c|c|c|c|c|c|c|c|}
\hline Parents & $\begin{array}{l}\text { Plant } \\
\text { height } \\
(\mathrm{cm})\end{array}$ & $\begin{array}{l}\text { Point to } \\
\text { first branch } \\
\text { (cm) }\end{array}$ & $\begin{array}{l}\text { Primary } \\
\text { branches/ } \\
\text { plant }\end{array}$ & $\begin{array}{l}\text { Secondary } \\
\text { branches/ } \\
\text { plant }\end{array}$ & $\begin{array}{l}\text { Main } \\
\text { shoot } \\
\text { length } \\
\text { (cm) }\end{array}$ & $\begin{array}{l}\text { Point to first } \\
\text { siliqua on } \\
\text { main shoot } \\
\text { (cm) }\end{array}$ & $\begin{array}{l}\text { Number of } \\
\text { siliquae on } \\
\text { main shoot }\end{array}$ & $\begin{array}{l}\text { Total } \\
\text { siliquae/ } \\
\text { plant }\end{array}$ & $\begin{array}{l}\text { Siliqua } \\
\text { length } \\
\text { (cm) }\end{array}$ & $\begin{array}{l}\text { Seeds/ } \\
\text { Siliqua }\end{array}$ & $\begin{array}{c}\text { Day to } \\
50 \% \\
\text { flowering }\end{array}$ & $\begin{array}{l}1,000 \text {-seed } \\
\text { weight }(\mathrm{g})\end{array}$ & $\begin{array}{c}\text { Seed } \\
\text { yield/ } \\
\text { plant (g) }\end{array}$ & $\begin{array}{l}\text { Biological } \\
\text { yield/ plant } \\
\text { (g) }\end{array}$ & $\begin{array}{l}\text { Harvest } \\
\text { index }\end{array}$ & $\begin{array}{l}\text { Seed } \\
\text { yield } \\
\text { (kg/ha) }\end{array}$ & $\begin{array}{l}\text { Oil } \\
\text { content } \\
(\%)\end{array}$ \\
\hline \multirow[t]{2}{*}{ CDL102 } & $-14.2^{\star \star}$ & $-14.63^{\star \star}$ & -0.13 & $2.9^{\star \star}$ & 1.32 & 0.05 & -4.69 & $71.12^{\star \star}$ & $0.42^{\star \star}$ & $2.12^{\star \star}$ & $-4.3^{\star *}$ & -0.25 & -2.47 & -19.53 & $0.63^{\star \star}$ & -222.3 & -1.83 \\
\hline & $\left(-12^{\star \star}\right)$ & $\left(-13.2^{\star \star}\right)$ & $(-0.87)$ & $(-0.54)$ & $\left(5.4^{\star \star}\right)$ & $\left(-1.1^{\star \star}\right)$ & $(-2.29)$ & $\left(32.5^{\star \star}\right)$ & $\left(0.41^{\star \star}\right)$ & $\left(1.91^{\star \star}\right)$ & $\left(-2.9^{\star \star}\right)$ & $(-0.1)$ & $(-3.04)$ & $\left(5.69^{\star \star}\right)$ & $(-2.8)$ & $(-120.5)$ & $(-1.8)$ \\
\hline \multirow[t]{2}{*}{ CDL161 } & $-7.5^{\star \star}$ & -1.03 & -0.56 & -0.1 & $1.87^{\star}$ & $-0.56^{\star *}$ & 0.45 & -18.66 & 0.08 & 0.14 & $-4.12^{\star \star}$ & $0.22^{\star \star}$ & $3.38^{\star *}$ & $19.2^{\star \star}$ & -0.52 & $267.1^{\star \star}$ & -0.11 \\
\hline & $(-3.3)$ & $\left(-1.87^{\star \star}\right)$ & $(0.27)$ & $\left(0.97^{\star \star}\right)$ & $\left(3.1^{\star \star}\right)$ & $\left(-2.4^{\star \star}\right)$ & $\left(3.67^{\star \star}\right)$ & $\left(40.6^{\star \star}\right)$ & $(-0.17)$ & $(-0.28)$ & $\left(-2.6^{\star \star}\right)$ & $\left(0.43^{\star \star}\right)$ & $\left(3.6^{\star \star}\right)$ & $\left(21.5^{\star \star}\right)$ & $(-0.19)$ & $\left(72.5^{\star \star}\right)$ & $(0.35)$ \\
\hline \multirow[t]{2}{*}{ CDL128 } & 1.75 & -1.25 & -0.18 & -0.48 & -1.29 & $-0.86^{\star \star}$ & -0.43 & 8.36 & 0.05 & -0.53 & $-2.2^{\star \star}$ & -0.26 & $3.5^{\star \star}$ & $4.35^{*}$ & $1.16^{\star \star}$ & 40.9 & $1.87^{\star \star}$ \\
\hline & (1.5) & $\left(-1.76^{\star \star}\right)$ & $(-0.39)$ & $(-0.05)$ & $(-2.3)$ & $(0.52)$ & $(-5.83)$ & $(-69.08)$ & $\left(0.17^{\star \star}\right)$ & $\left(0.54^{\star \star}\right)$ & $\left(-2.4^{\star \star}\right)$ & $(0.11)$ & $\left(3.92^{\star \star}\right)$ & $(1.7)$ & $\left(2.1^{\star \star}\right)$ & $(-142.7)$ & $\left(2^{\star \star}\right)$ \\
\hline CDL141 & 5.44 & 2.82 & $0.81^{\star \star}$ & -0.01 & -4.68 & $-0.26^{\star \star}$ & 0.22 & -9.14 & -0.2 & -0.74 & 1.94 & -0.02 & $0.99^{*}$ & -1.77 & $0.47^{\star \star}$ & $97.8^{\star \star}$ & $1.51^{\star \star}$ \\
\hline \multirow{3}{*}{ CDL112 } & (8.84) & (6.9) & (0.03) & $(-0.89)$ & $(-6.14)$ & (1.66) & $(-2.97)$ & $(-112.47)$ & $(-0.15)$ & $(-0.51)$ & $(2.71)$ & $(-0.31)$ & $(-5.76)$ & $(-29.34)$ & $(-0.37)$ & $(-265.8)$ & $\left(0.5^{\star}\right)$ \\
\hline & 18.81 & -0.6 & $0.39^{\star \star}$ & $1.42^{\star \star}$ & $3.31^{\star *}$ & $-1.33^{\star \star}$ & $5.42^{\star \star}$ & $51.38^{\star \star}$ & -0.35 & -0.14 & 3.01 & -0.14 & $4.76^{\star \star}$ & $30.13^{\star \star}$ & -0.76 & $83.2^{\star \star}$ & $1.5^{\star \star}$ \\
\hline & (18.27) & (7.41) & $(0.21)$ & $(0.14)$ & $\left(4.5^{\star \star}\right)$ & $\left(-0.92^{\star \star}\right)$ & $\left(3.27^{\star \star}\right)$ & $\left(80.5^{\star \star}\right)$ & $(-0.36)$ & $(-0.37)$ & $(4.73)$ & $(-0.33)$ & $(0.45)$ & $\left(19.1^{\star \star}\right)$ & $(-1.87)$ & $\left(164^{\star \star}\right)$ & $(-0.24)$ \\
\hline \multirow[t]{2}{*}{ CDL186 } & 9.21 & 10.07 & $0.49^{* \star}$ & -0.22 & -1.25 & 0.23 & $2.33^{*}$ & -32.96 & -0.27 & -0.13 & 2.07 & $0.23^{\star \star}$ & $1.29^{\star \star}$ & 2.26 & $0.77^{\star \star}$ & $208.4^{\star \star}$ & -0.1 \\
\hline & $(9.07)$ & $(3.41)$ & $\left(0.8^{\star \star}\right)$ & $\left(1.31^{\star \star}\right)$ & $(-3.78)$ & $\left(-1.15^{\star \star}\right)$ & $(-0.28)$ & $(-18.8)$ & (0) & $\left(0.51^{\star \star}\right)$ & (3.55) & $\left(0.2^{\star \star}\right)$ & $(-0.3)$ & (3.05) & $(-0.97)$ & $\left(54.9^{\star}\right)$ & $(-0.5)$ \\
\hline \multirow[t]{2}{*}{ CDL182 } & 4.58 & 8.6 & $0.34^{\star \star}$ & -0.61 & -1.35 & -0.01 & -1.2 & -71.05 & 0 & 0.30 & 1.25 & 0.07 & -0.84 & -7.1 & $0.64^{\star \star}$ & -24.18 & -0.8 \\
\hline & (3.52) & $(9.07)$ & $(0.05)$ & $(-1.37)$ & $(-1.87)$ & $\left(-1.35^{\star \star}\right)$ & $(-1.78)$ & $(-62.07)$ & $(-0.11)$ & $\left(0.45^{\star}\right)$ & (1.82) & $\left(0.18^{\star \star}\right)$ & $\left(3.04^{\star \star}\right)$ & $\left(5.78^{\star \star}\right)$ & $\left(1.1^{\star \star}\right)$ & $(-66.9)$ & $(-0.2)$ \\
\hline \multirow[t]{2}{*}{ CDL121 } & 2.85 & 3.33 & -0.12 & -0.08 & -0.6 & 0.29 & -0.07 & -27.22 & -0.41 & -1.87 & 1.25 & $0.16^{\star \star}$ & -1.92 & -2.22 & -0.61 & -186 & -0.88 \\
\hline & $(5.61)$ & (9.56) & $(-0.32)$ & $(-0.73)$ & $(1.26)$ & $(0.94)$ & $\left(1.85^{\star}\right)$ & $(-49.1)$ & $(-0.37)$ & $(-1.77)$ & (2.57) & $\left(0.2^{\star \star}\right)$ & $(-0.71)$ & $(-6.54)$ & $\left(0.5^{\star \star}\right)$ & $(-4.58)$ & $(0.04)$ \\
\hline \multirow[t]{2}{*}{ CDL103 } & $-6.02^{\star \star}$ & -1.03 & -0.52 & -1.19 & 0.54 & 0.35 & -1.52 & -35.57 & $0.16^{\star \star}$ & $0.71^{\star \star}$ & $-0.8^{\star \star}$ & 0.05 & -4.59 & -14.92 & -1.22 & -524.9 & -1.65 \\
\hline & $\left(-7.2^{\star \star}\right)$ & $\left(-5.11^{\star \star}\right)$ & $(-0.35)$ & $(-0.92)$ & $\left(3.3^{\star \star}\right)$ & (2.16) & $(-0.99)$ & $(-48.38)$ & $\left(0.12^{\star \star}\right)$ & $\left(0.76^{\star \star}\right)$ & $\left(-1.5^{\star \star}\right)$ & $\left(0.15^{\star \star}\right)$ & $(-1.71)$ & $(-7.07)$ & $(-0.12)$ & $(-307.1)$ & $(-0.4)$ \\
\hline \multirow[t]{2}{*}{ CDL101 } & 0.12 & 4.81 & $0.53^{\star \star}$ & -0.13 & -3.04 & -0.11 & 1.75 & -10.94 & $0.12^{\star \star}$ & 0.22 & 1.25 & -0.21 & -1.04 & -8.75 & $0.55^{\star \star}$ & $164.9^{\star \star}$ & $1.8^{\star \star}$ \\
\hline & $(-2.19)$ & (3.94) & $\left(0.5^{\star \star}\right)$ & $(-0.27)$ & $(-2.97)$ & $\left(-1.83^{\star \star}\right)$ & $\left(5.58^{\star \star}\right)$ & $\left(11.45^{\star}\right)$ & $(0.07)$ & $(-0.27)$ & (1.79) & $(-0.13)$ & $\left(1.4^{*}\right)$ & $(-4.6)$ & $\left(1.7^{\star \star}\right)$ & $\left(333^{\star \star}\right)$ & $\left(1.2^{\star \star}\right)$ \\
\hline \multirow[t]{2}{*}{ CDL25 } & 4.64 & 3.51 & -0.2 & $0.43^{*}$ & $4.13^{\star \star}$ & 1.07 & $5.65^{\star \star}$ & $34.03^{\star \star}$ & -0.41 & -0.75 & 0.74 & -0.24 & $2.04^{\star \star}$ & 3.22 & $1.59^{\star \star}$ & $259.3^{\star \star}$ & 0.4 \\
\hline & $(4.09)$ & $\left(-1.25^{\star \star}\right)$ & $(-0.33)$ & (0.34) & $(1.21)$ & $(0.61)$ & $\left(5.58^{\star \star}\right)$ & $\left(86.6^{\star \star}\right)$ & $(-0.22)$ & $(-0.13)$ & $(-0.22)$ & $(-0.23)$ & $\left(5.68^{\star \star}\right)$ & $\left(15.1^{\star \star}\right)$ & $\left(1.9^{\star \star}\right)$ & $\left(313^{\star \star}\right)$ & $\left(1.0^{\star \star}\right)$ \\
\hline \multirow[t]{2}{*}{ CDL89 } & $-4.25^{\star}$ & $-2.62^{\star \star}$ & 0.15 & 0.07 & -3.95 & $-0.79^{\star \star}$ & 0.15 & 6.04 & -0.12 & -1.19 & 1.74 & $0.31^{\star *}$ & $0.91^{\star}$ & 0.41 & $0.36^{\star}$ & $221.4^{\star \star}$ & $1.25^{\star \star}$ \\
\hline & $(-3.02)$ & $(2.25)$ & $\left(0.5^{\star \star}\right)$ & $(0.38)$ & $(-3.84)$ & $\left(-0.85^{\star \star}\right)$ & $\left(2.69^{\star \star}\right)$ & $(-18.41)$ & $(-0.12)$ & $(-1.16)$ & (2.96) & $\left(0.11^{\star}\right)$ & $(-0.76)$ & $(-12.6)$ & $\left(1.4^{\star \star}\right)$ & $\left(205^{\star \star}\right)$ & $\left(0.6^{\star}\right)$ \\
\hline \multirow[t]{2}{*}{ CDL104 } & $-6.5^{\star \star}$ & $-2.04^{\star \star}$ & -0.22 & -0.04 & -1.48 & $-0.46^{\star *}$ & -2.61 & $36.33^{\star \star}$ & $0.11^{\star}$ & 0.01 & 1.43 & -0.07 & -0.89 & 0.75 & -0.61 & 40.56 & -0.5 \\
\hline & $\left(-8^{\star \star}\right)$ & $\left(-5^{\star \star}\right)$ & $(0.26)$ & $\left(0.89^{\star \star}\right)$ & $(-3.21)$ & $\left(-1.46^{\star \star}\right)$ & $(-3.06)$ & $\left(43.8^{\star \star}\right)$ & $\left(0.11^{\star \star}\right)$ & $(-0.3)$ & $\left(-1.9^{\star \star}\right)$ & $(-0.22)$ & $(-1.45)$ & (1.63) & $(-1)$ & (35.9) & $(-0.9)$ \\
\hline \multirow[t]{2}{*}{ CDL105 } & $-9.02^{\star \star}$ & $-6.56^{\star \star}$ & -0.57 & -1.3 & $3.31^{\star \star}$ & $0.94^{\star \star}$ & -2.69 & -6.47 & $0.59^{\star \star}$ & $1.56^{\star \star}$ & $-1.61^{\star \star}$ & -0.06 & -3.97 & 1.14 & -2.47 & -458.4 & -0.96 \\
\hline & $\left(-13^{\star \star}\right)$ & $\left(-9.79^{\star \star}\right)$ & $(-0.59)$ & $(-1.11)$ & (1.18) & $(0.74)$ & $(-5.23)$ & $(-14.96)$ & $\left(0.49^{\star \star}\right)$ & $\left(0.72^{\star \star}\right)$ & $\left(-5.1^{\star \star}\right)$ & $(-0.16)$ & $(-5.46)$ & $(-19.63)$ & $(-1.2)$ & $(-332.4)$ & $(-0.79)$ \\
\hline \multirow[t]{2}{*}{ CDL106 } & 0.13 & $-3.38^{\star \star}$ & -0.22 & -0.66 & $3.18^{\star \star}$ & 1.46 & -2.75 & 4.74 & $0.21^{\star \star}$ & 0.28 & $-1.61^{\star \star}$ & $0.19^{\star \star}$ & -1.13 & -7.17 & 0.02 & 32.1 & -1.31 \\
\hline & $(-2.19)$ & $\left(-4.52^{\star \star}\right)$ & $(0.18)$ & $\left(1.84^{\star \star}\right)$ & $\left(4.2^{\star \star}\right)$ & (0.6) & $(-0.22)$ & $\left(97.7^{\star \star}\right)$ & $\left(0.11^{\star \star}\right)$ & $(-0.09)$ & $\left(-3.4^{\star \star}\right)$ & $\left(0.09^{\star}\right)$ & $\left(1.12^{\star}\right)$ & $\left(6.29^{\star \star}\right)$ & $(-0.23)$ & $\left(60.9^{\star}\right)$ & $(-0.91)$ \\
\hline \multirow[t]{2}{*}{ SE } & 1.98 & 0.64 & 0.08 & 0.17 & 0.72 & 0.07 & 0.82 & 3.97 & 0.04 & 0.16 & 0.42 & 0.05 & 0.42 & 1.65 & 0.14 & 27.01 & 0.20 \\
\hline & (1.6) & (0.32) & $(0.13)$ & $(0.18)$ & $(0.83)$ & $(0.12)$ & $(0.84)$ & (5.14) & $(0.03)$ & $(0.17)$ & $(0.48)$ & $(0.04)$ & $(0.48)$ & $(1.84)$ & $(0.16)$ & (26.72) & $(0.21)$ \\
\hline \multirow[t]{2}{*}{$\mathrm{CD}(\mathrm{P}=0.05)$} & 4.24 & 1.36 & 0.16 & 0.36 & 1.55 & 0.16 & 1.75 & 8.51 & 0.08 & 0.35 & 0.91 & 0.11 & 0.89 & 3.55 & 0.29 & 53.2 & 0.43 \\
\hline & $(3.44)$ & (0.69) & $(0.28)$ & $(0.38)$ & $(1.78)$ & $(0.26)$ & $(1.8)$ & (11.02) & $(0.07)$ & $(0.36)$ & (1.03) & $(0.08)$ & (1.03) & (3.94) & $(0.33)$ & (52.63) & $(0.45)$ \\
\hline \multirow[t]{2}{*}{$C D(P=0.01)$} & 5.88 & 1.89 & 0.23 & 0.50 & 2.15 & 0.22 & 2.43 & 11.81 & 0.11 & 0.49 & 1.26 & 0.15 & 1.23 & 4.92 & 0.40 & 70.13 & 0.60 \\
\hline & $(4.77)$ & $(0.96)$ & $(0.39)$ & $(0.53)$ & $(2.48)$ & $(0.36)$ & (2.49) & (15.3) & $(0.10)$ & $(0.50)$ & (1.44) & $(0.11)$ & (1.43) & (5.46) & $(0.46)$ & (69.38) & $(0.63)$ \\
\hline
\end{tabular}


TABLE 5 | Estimates of heterobeltiosis and specific combining ability (SCA) effects of 105 hybrids of CDLs for seed yield (kg/ha) under irrigated and rainfed conditions.

\begin{tabular}{|c|c|c|c|c|c|c|c|c|c|c|c|c|c|c|c|}
\hline & CDL102 & CDL161 & CDL128 & CDL141 & CDL112 & CDL186 & CDL182 & CDL121 & CDL103 & CDL101 & CDL25 & CDL89 & CDL104 & CDL105 & CDL106 \\
\hline \multirow[t]{2}{*}{ CDL102 } & & -3.36 & -27.74 & -4.26 & -13.98 & $42.82^{\star \star}$ & -36.94 & -40.92 & -8.82 & 20.32 & $46.03^{\star \star}$ & $51.62^{\star \star}$ & -15.33 & -19.52 & $55.86^{\star \star}$ \\
\hline & & $(-297.5)$ & $(-532.5)$ & $(-322.8)$ & $(-235.9)$ & $\left(605.6^{\star \star}\right)$ & $(-970.1)$ & $(-1000)$ & (105.6) & $\left(626.9^{\star \star}\right)$ & $\left(743.6^{\star \star}\right)$ & $\left(988.7^{\star \star}\right)$ & $(-615.4)$ & $(-21$ & $\left(1093^{\star \star}\right)$ \\
\hline \multirow[t]{2}{*}{ CDL161 } & $45^{\star \star}$ & & $34.03^{\star \star}$ & $43.07^{\star \star}$ & -9.87 & $39.72^{\star \star}$ & $12.32^{\star}$ & 8.98 & $12.95^{\star}$ & $40.04^{\star \star}$ & -3.36 & $29.75^{\star \star}$ & -0.35 & $22.99^{\star \star}$ & $14.67^{\star}$ \\
\hline & $\left(685^{\star \star}\right)$ & & $\left(778.2^{\star \star}\right)$ & $\left(615.7^{\star \star}\right)$ & $(-608.6)$ & $\left(416.3^{\star \star}\right)$ & $(-78.9)$ & $(-6)$ & $\left(438.5^{\star \star}\right)$ & $\left(693^{\star \star}\right)$ & $(-779.1)$ & (138.3) & $(-480.4)$ & $\left(638.6^{\star \star}\right)$ & $(-73)$ \\
\hline \multirow[t]{2}{*}{ CDL128 } & $17.23^{\star}$ & $44.04^{\star \star}$ & & $15.69^{\star \star}$ & -38.8 & $20.11^{\star \star}$ & $32.6^{\star \star}$ & -31.93 & -2.7 & 6.86 & $12.01^{\star}$ & -2.8 & $10.96^{\star}$ & -28.98 & -27.36 \\
\hline & $\left(289^{\star \star}\right)$ & $\left(712.8^{\star \star}\right)$ & & $\left(412.9^{\star \star}\right)$ & $(-1160.2)$ & $\left(431.3^{\star \star}\right)$ & $\left(1027^{\star \star}\right)$ & $(-690.7)$ & $\left(500^{\star \star}\right)$ & (88.7) & (144.3) & $(-249.5)$ & $\left(332.5^{\star \star}\right)$ & $(-332.4)$ & $(-775.7)$ \\
\hline \multirow[t]{2}{*}{ CDL141 } & 0.5 & $82.34^{\star \star}$ & $31.22^{\star \star}$ & & $19.75^{\star \star}$ & $24.89^{\star \star}$ & $30.16^{\star \star}$ & $21.08^{\star \star}$ & $12.6^{\star}$ & -8.68 & $40.25^{\star \star}$ & 7.52 & $13.01^{\star}$ & -6.99 & $14.13^{\star}$ \\
\hline & (8.2) & $\left(788^{\star \star}\right)$ & $\left(756^{\star \star}\right)$ & & $\left(402.3^{\star \star}\right)$ & $(-31.2)$ & $\left(415.3^{\star \star}\right)$ & $\left(268.8^{\star \star}\right)$ & $\left(398^{\star \star}\right)$ & $(-510)$ & $\left(298^{\star \star}\right)$ & $(-438.7)$ & $(-157.8)$ & $(-154.4)$ & $(-121.6)$ \\
\hline \multirow[t]{2}{*}{ CDL112 } & $20.9^{\star \star}$ & 0.62 & -22.62 & -0.86 & & 1.76 & 7.33 & -0.1 & -2.05 & -9.97 & 7.41 & $52.49^{\star \star}$ & 8.5 & -4.11 & $17.3^{\star \star}$ \\
\hline & $\left(565^{\star \star}\right)$ & $(-178)$ & $(-593.3)$ & (120.1) & & $(-219.3)$ & (171.6) & (122.3) & $\left(406^{\star \star}\right)$ & $(-509.2)$ & $(-109.7)$ & $\left(1209^{\star \star}\right)$ & $(140.2)$ & $\left(280.8^{\star \star}\right)$ & $\left(398.7^{\star \star}\right)$ \\
\hline \multirow[t]{2}{*}{ CDL186 } & $31.68^{\star \star}$ & $53.41^{\star \star}$ & $23.27^{\star \star}$ & $17.23^{\star \star}$ & -15.56 & & -26.45 & $67.19^{\star \star}$ & -11.02 & $22.39^{\star \star}$ & $29.24^{\star \star}$ & $23.76^{\star \star}$ & $49.03^{\star \star}$ & $27.94^{\star \star}$ & $57.43^{\star \star}$ \\
\hline & $\left(399^{\star \star}\right)$ & $\left(481.7^{\star \star}\right)$ & $\left(252.5^{\star}\right)$ & (46.5) & $(-599.5)$ & & $(-1134.2)$ & $\left(994.4^{\star \star}\right)$ & $(-461.2)$ & $\left(254.5^{\star}\right)$ & $(-101)$ & $(-141.4)$ & $\left(351.1^{\star \star}\right)$ & $\left(366.2^{\star \star}\right)$ & $\left(552.4^{\star \star}\right)$ \\
\hline \multirow[t]{2}{*}{ CDL182 } & -25.76 & $27.3^{\star \star}$ & $26.66^{\star *}$ & $55.83^{\star \star}$ & 10.44 & 8.19 & & $69.03^{* *}$ & -6.12 & $12.03^{\star}$ & $18.91^{\star \star}$ & $33.01^{\star \star}$ & -10.38 & -31.04 & 4.92 \\
\hline & $(-789.5)$ & $(-396.4)$ & $\left(452.3^{\star \star}\right)$ & $\left(375^{\star \star}\right)$ & $\left(227.9^{\star}\right)$ & $(-345.7)$ & & $\left(1687^{\star \star}\right)$ & (115.9) & (195.4) & $(-32.3)$ & $\left(363.9^{\star \star}\right)$ & $(-558)$ & $(-584)$ & $(-160.6)$ \\
\hline \multirow[t]{2}{*}{ CDL121 } & -49.24 & 5.92 & -8.25 & -12.29 & $22.94^{\star \star}$ & $49.45^{\star \star}$ & $48.49^{\star \star}$ & & $66.24^{\star \star}$ & -28.21 & $45.92^{\star \star}$ & 5.86 & $31.69^{\star *}$ & $22.45^{\star \star}$ & $69.56^{\star \star}$ \\
\hline & $(-1282.4)$ & $(-103.2)$ & $(-240.5)$ & $(-217.9)$ & $\left(504.5^{\star \star}\right)$ & $\left(997.5^{\star \star}\right)$ & $\left(1095^{\star \star}\right)$ & & $\left(394.4^{\star \star}\right)$ & $(-776.1)$ & $\left(705^{\star \star}\right)$ & $(-196.4)$ & $(-76.7)$ & $\left(294.5^{\star \star}\right)$ & (163.5) \\
\hline \multirow[t]{2}{*}{ CDL103 } & -8.83 & $31.02^{\star *}$ & -12.24 & $35.44^{\star \star}$ & -14.75 & -17.28 & $60.94^{\star \star}$ & $21.44^{\star \star}$ & & -34.08 & $13.37^{*}$ & -28.3 & -42.11 & $36.64^{\star \star}$ & $48.02^{\star \star}$ \\
\hline & $(-163.2)$ & $(-89.5)$ & $(-202.1)$ & $\left(237.5^{\star}\right)$ & $(-215.2)$ & $(-649.9)$ & $\left(505.3^{\star \star}\right)$ & $\left(662.5^{\star \star}\right)$ & & $(-602.7)$ & $\left(241.3^{\star}\right)$ & $(-715.3)$ & $(-1193.3)$ & $\left(919.5^{\star \star}\right)$ & (142.9) \\
\hline \multirow[t]{2}{*}{ CDL101 } & 1.11 & $25.21^{\star \star}$ & -5.43 & 0.8 & 5.82 & $42.82^{\star \star}$ & 4.42 & -5.83 & $18.1^{\star \star}$ & & 7.79 & $12.49^{\star}$ & $42.31^{\star \star}$ & -14.79 & $28.11^{\star \star}$ \\
\hline & $(-89.5)$ & $\left(383.6^{\star \star}\right)$ & $(-247.8)$ & (47.3) & $(-244.2)$ & $\left(887.7^{\star \star}\right)$ & $(-51.6)$ & $(-397.1)$ & $\left(566.6^{\star \star}\right)$ & & $(-207.5)$ & $(-37.4)$ & $\left(983.5^{\star \star}\right)$ & $(-125.9)$ & $\left(591.9^{\star \star}\right)$ \\
\hline \multirow[t]{2}{*}{ CDL25 } & $37.81^{* \star}$ & $15.18^{\star \star}$ & -5.72 & 0.59 & 6.94 & -6.95 & -15.19 & 11.41 & & 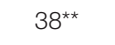 & & & $71.06^{\star \star}$ & -1.75 & $.44^{\star \star}$ \\
\hline & $\left(995^{\star \star}\right)$ & (168.5) & $(-201.4)$ & (98.3) & $(-154.4)$ & $(-433.6)$ & $(-542.3)$ & (140) & $\left(437^{\star \star}\right)$ & $\left(546.7^{\star \star}\right)$ & & $(-88.1)$ & $\left(1098^{\star \star}\right)$ & $(-198.1)$ & .9) \\
\hline \multirow[t]{2}{*}{ CDL89 } & 4.09 & -12.61 & -34.65 & -43.03 & $31.22^{\star \star}$ & 8.61 & -12.29 & -22.27 & -13.66 & -23.92 & -3.97 & & $58.19^{\star \star}$ & -16.7 & \\
\hline & $\left(569^{\star \star}\right)$ & $(-157.7)$ & $(-646.3)$ & $(-790.7)$ & $\left(1150^{\star \star}\right)$ & $\left(537.5^{\star \star}\right)$ & $(-7.9)$ & $(-388.9)$ & (188.6) & $(-779.3)$ & $(-121.8)$ & & $\left(891^{\star \star}\right)$ & $(-490.7)$ & $\left(269^{\star \star}\right)$ \\
\hline \multirow[t]{2}{*}{ CDL104 } & -31.15 & -11.23 & -1.19 & -22.57 & -15.56 & -2.78 & $27.42^{\star \star}$ & 8.63 & -25.59 & $17.69^{\star \star}$ & $16.57^{\star \star}$ & 5.46 & & $23.97^{\star \star}$ & $99.55^{\star \star}$ \\
\hline & $(-774.8)$ & $(-443.8)$ & (35.4) & $(-404)$ & $(-580.5)$ & $(-204.2)$ & $\left(712.1^{\star \star}\right)$ & (155.5) & $(-441.9)$ & $\left(212.3^{\star}\right)$ & $\left(243.9^{\star}\right)$ & $\left(455.9^{\star \star}\right)$ & & (98.5) & $\left(1043^{\star \star}\right)$ \\
\hline \multirow[t]{2}{*}{ CDL105 } & -45.27 & -3.1 & -21.64 & -24.97 & 2.56 & -19.94 & -29.8 & -22.11 & -29.63 & -27.71 & -10.82 & -27.14 & -35.78 & & -17.25 \\
\hline & $(-615.7)$ & $\left(425.6^{\star \star}\right)$ & $(98.2)$ & (123.8) & $\left(499^{\star \star}\right)$ & $(-49.7)$ & $(-216.5)$ & $(-53.9)$ & $(28.7)$ & $(-555.4)$ & $(-40.5)$ & $(-216.7)$ & $(-494.4)$ & & $(-724.3)$ \\
\hline \multirow[t]{2}{*}{ CDL106 } & $36.47^{\star \star}$ & $26.96^{\star \star}$ & -0.28 & 3.82 & 9.42 & $28.72^{\star \star}$ & $59.53^{\star \star}$ & -11.5 & $25.87^{\star \star}$ & $42.33^{\star \star}$ & 3.67 & -8.52 & $71.83^{\star \star}$ & -44.51 & \\
\hline & $\left(502^{\star \star}\right)$ & $(-227.1)$ & $(-295.1)$ & $-358.4)$ & (72.3) & $(-34.7)$ & $\left(573.4^{\star \star}\right)$ & $(-525)$ & (130.3) & $\left(867.9^{\star \star}\right)$ & $(-142.2)$ & $(-15.6)$ & $\left(1752^{\star \star}\right)$ & $(-775)$ & \\
\hline
\end{tabular}

Heterobeltiosis and SCA effects for rainfed (above diagonal) and irrigated condition (below diagonal) Values in parenthesis represent SCA effects.

${ }^{* *}$ Significant at $p=0.01$ and ${ }^{*}$ significant $p=0.01$ and ${ }^{*}$ significant at $P=0.05$.

irrigated condition $\left(\mathrm{Y}_{I R R}\right)$, seed yield under rainfed condition $\left(\mathrm{Y}_{R F}\right), \mathrm{WUE}_{R F}, \mathrm{WUE}_{I R R}, \mathrm{DSI}, \mathrm{DTI}, \mathrm{TOL}$, and MRP were calculated. Yield under rainfed condition $\left(\mathrm{Y}_{R F}\right)$ were showing significant and positive association with $\mathrm{WUE}_{R F}, \mathrm{WUE}_{I R}$, DTI, MRP, and TOL. Similarly, significantly positive association of $\mathrm{Y}_{I R}$ with DTI and MRP was observed (Supplementary Table 2). WUE $_{R F}$ exhibited a positive and significant correlation with DSI, DTI, TOL, and MRP, while $\mathrm{WUE}_{I R}$ showed a positive and significant correlation with DTI and MRP. DTI and MRP were identified to be significantly associated with all the traits except DSI, thus, indicating the usefulness of these two indices in indirect selection for drought tolerance.

The WUE $\left(\mathrm{kg} \mathrm{m}^{-3}\right)$ was calculated for all hybrids along with parental CDLs both under rainfed and irrigated conditions. WUE among the parental lines raised under rainfed condition varied from 1.27 to $2.59 \mathrm{~kg} \mathrm{~m}^{-3}$ and recorded the highest and lowest values for CDL128 and CDL103, respectively (Table 8). Under irrigated condition it ranged from 0.73 to $1.50 \mathrm{~kg} \mathrm{~m}^{-3}$ with the highest and lowest values for CDL89 and CDL103, respectively. Among 105 hybrids evaluated under rainfed condition, WUE varied from 1.02 to $3.86 \mathrm{~kg} \mathrm{~m}^{-3}$ and the highest value was recorded for the cross CDL112 $\times$ CDL89. On the other hand, WUE varied from 0.59 to $2.13 \mathrm{~kg} \mathrm{~m}^{-3}$ under the irrigated condition and exhibited the highest value for CDL104 $\times$ CDL106 cross (Table 8). In general, the hybrids observed higher WUE than their parental CDLs. Overall, the mean of seed yield (kg/ha) and WUE of parental CDLs and hybrids was recorded higher under rainfed condition than in irrigated condition (Figure 1).

Further, drought tolerance index (DTI) and mean relative performance (MRP) were calculated from average values of individual parent and hybrid for all the 17 traits. All the traits, except point to first branch and point to first siliqua on the main shoot, exhibited higher DTI and MRP for hybrids than their corresponding parents (Figure 2). DTI for seed yield (kg/ha) varied from 0.25 to 2.55 in hybrids, whereas it varied from 0.31 to 1.12 in CDLs (Table 8). Some of the hybrids such as CDL112 $\times$ CDL89 (DTI: 2.55), CDL104 $\times$ CDL106 (DTI: 2.49) CDL182 × CDL121 (DTI: 2.23), CDL186 × CDL121 (DTI: 2.0), CDL101 $\times$ CDL106 (DTI: 1.99), CDL102 $\times$ CDL25 (DTI: 1.95$)$, CDL25 × CDL104 (DTI: 1.93), CDL161 × CDL101 (DTI: 1.91), 
TABLE 6 | Combining ability and heterobeltiosis of hybrids derived from CDLs with different levels of GCA effects for seed yield (kg/ha) under irrigated and rainfed conditions.

\begin{tabular}{|c|c|c|c|c|c|}
\hline \multirow[t]{2}{*}{ Environment } & \multicolumn{2}{|c|}{ GCA effects of CDLs for Seed yield (kg/ha) } & \multicolumn{3}{|c|}{ Hybrids with highly significant SCA effects and $>15 \%$ heterobeltiosis } \\
\hline & Significant & Non-significant & $\begin{array}{l}\text { Hybrids developed from } \\
\text { crossing CDLs with } \\
\text { significant GCA effects }\end{array}$ & $\begin{array}{l}\text { Hybrids developed from } \\
\text { crossing CDLs with significant } \\
\text { and non-significant } \\
\text { GCA effects }\end{array}$ & $\begin{array}{l}\text { Hybrids developed from } \\
\text { crossing CDLs with } \\
\text { non-significant } \\
\text { GCA effects }\end{array}$ \\
\hline $\begin{array}{l}\text { Rainfed condition } \\
\text { (RF) }\end{array}$ & $\begin{array}{l}\text { CDL161, CDL141, } \\
\text { CDL112, CDL186, } \\
\text { CDL101, CDL25 } \\
\text { and CDL89 }\end{array}$ & $\begin{array}{l}\text { CDL102, CDL128, } \\
\text { CDL182, CDL121, } \\
\text { CDL103, CDL104, } \\
\text { CDL105 and } \\
\text { CDL106 }\end{array}$ & $\begin{array}{l}\text { CDL161 × CDL141, } \\
\text { CDL161 × CDL186, } \\
\text { CDL161 × CDL101, } \\
\text { CDL141 × CDL112, } \\
\text { CDL141 × CDL25, } \\
\text { CDL112 × CDL89, } \\
\text { CDL186 × CDL101 } \\
(7 \text { Hybrids) }\end{array}$ & $\begin{array}{l}\text { CDL161 × CDL128, } \\
\text { CDL161 × CDL105, } \\
\text { CDL141 × CDL128, } \\
\text { CDL141 × CDL182, } \\
\text { CDL141 × CDL121, } \\
\text { CDL112 × CDL106, } \\
\text { CDL186 × CDL102, } \\
\text { CDL186 × CDL128, } \\
\text { CDL186 × CDL121, } \\
\text { CDL186 × CDL104, } \\
\text { CDL186 × CDL105, } \\
\text { CDL186 × CDL106, } \\
\text { CDL101 × CDL102, } \\
\text { CDL101 × CDL104, } \\
\text { CDL101 × CDL106, } \\
\text { CDL25 } \times \text { CDL102, } \\
\text { CDL25 } \times \text { CDL121, } \\
\text { CDL25 } \times \text { CDL104, } \\
\text { CDL89 } \times \text { CDL102, } \\
\text { CDL89 } \times \text { CDL182, } \\
\text { CDL89 } \times \text { CDL104, } \\
\text { CDL89 } \times \text { CDL106 } \\
(22 H y b i d s)\end{array}$ & $\begin{array}{l}\text { CDL102 × CDL106, } \\
\text { CDL128 × CDL182, } \\
\text { CDL182 × CDL121, } \\
\text { CDL121 × CDL103, } \\
\text { CDL121 × CDL105, } \\
\text { CDL103 × CDL105, } \\
\text { CDL104 × CDL106 } \\
(7 \text { Hybrids) }\end{array}$ \\
\hline $\begin{array}{l}\text { Irrigated condition } \\
(\mathrm{IR})\end{array}$ & $\begin{array}{l}\text { CDL161, CDL112, } \\
\text { CDL186, CDL101, } \\
\text { CDL25, CDL89, } \\
\text { and CDL106 }\end{array}$ & $\begin{array}{l}\text { CDL102, CDL128, } \\
\text { CDL141, CDL182, } \\
\text { CDL121, CDL103, } \\
\text { CDL104, and } \\
\text { CDL105 }\end{array}$ & $\begin{array}{l}\text { CDL161 × CDL101, } \\
\text { CDL161 × CDL186, } \\
\text { CDL112 × CDL89, } \\
\text { CDL101 × CDL25, } \\
\text { CDL186 × CDL101, } \\
\text { CDL101 × CDL106 } \\
(6 \text { Hybrids) }\end{array}$ & $\begin{array}{l}\text { CDL161 } \times \text { CDL102, } \\
\text { CDL161 } \times \text { CDL128, } \\
\text { CDL161 } \times \text { CDL141 } \\
\text { CDL112 × CDL102, } \\
\text { CDL112 × CDL121, } \\
\text { CDL101 × CDL103, } \\
\text { CDL101 × CDL104, } \\
\text { CDL25 } \times \text { CDL102, } \\
\text { CDL25 } \times \text { CDL104, } \\
\text { CDL186 } \times \text { CDL102, } \\
\text { CDL186 × CDL128, } \\
\text { CDL186 } \times \text { CDL121, } \\
\text { CDL106 } \times \text { CDL102, } \\
\text { CDL106 } \times \text { CDL182, } \\
\text { CDL106 } \times \text { CDL104 }(15 \text { Hybrids })\end{array}$ & $\begin{array}{l}\text { CDL102 × CDL128, } \\
\text { CDL128 × CDL141, } \\
\text { CDL128 × CDL182, } \\
\text { CDL141 × CDL182, } \\
\text { CDL141 × CDL103, } \\
\text { CDL182 × CDL121, } \\
\text { CDL182 × CDL103, } \\
\text { CDL182 × CDL104, } \\
\text { CDL121 × CDL103 } \\
(9 \text { Hybrids) }\end{array}$ \\
\hline $\begin{array}{l}\text { Number common } \\
\text { in RF and IR } \\
\text { conditions }\end{array}$ & 6 CDLs & 7 CDLs & 4 hybrids & 7 hybrids & 3 hybrids \\
\hline
\end{tabular}

and CDL186 $\times$ CDL101 (DTI: 1.91$)$ has a DTI value of $>1$, indicating their superiority under both moisture-stress and nonstress conditions. Among the CDLs highest MRP was recorded for CDL89 (2.09) and the lowest was observed for CDL103 (1.09). Among the hybrids, CDL112 $\times$ CDL89 recorded with the highest (3.11) MRP (Table 8). Further, nineteen hybrids, viz., CDL102 × CDL186, CDL102 × CDL25, CDL102 × CDL89, CDL102 × CDL106, CDL161 × CDL128, CDL161 × CDL141, CDL161 × CDL186, CDL161 × CDL101, CDL128 × CDL141, CDL186 × CDL121, CDL186 × CDL101, CDL182 × CDL121, CDL121 × CDL25, CDL101 × CDL104, CDL101 × CDL106, CDL25 × CDL89, CDL25 × CDL104, CDL89 $\times$ CDL104, and CDL104 $\times$ CDL106 were identified to be significantly better in productivity and possessing higher values of DTI and MRP (Table 8).

\section{DISCUSSION}

The present study revealed the usefulness of CDLs for improving heterosis and WUE in B. juncea. The existence of significant differences $(p<0.001)$ among parental CDLs and hybrids for all the 17 traits studied under irrigated and rainfed conditions indicated wide variability for the test traits in parents and their hybrids. The significance of mean squares among parents and hybrids suggested the suitability of the CDLs for combining 
TABLE 7 | Mean values recorded on 15 parents and 105 hybrids for different traits under irrigated and rainfed conditions.

\begin{tabular}{|c|c|c|c|c|}
\hline \multirow[t]{2}{*}{ Traits } & \multicolumn{2}{|c|}{ Parents } & \multicolumn{2}{|c|}{ Hybrids } \\
\hline & IR & $\mathbf{R F}$ & IR & $\mathbf{R F}$ \\
\hline Plant height (cm) & $217.91+$ & 216.87 & $231.81^{*}$ & 229.88 \\
\hline Point to first branch (cm) & 34.08 & $52.61+$ & 32.09 & $47.86^{*}$ \\
\hline Primary branches/plant & $7.02+$ & 6.49 & $7.68^{\star}$ & 6.96 \\
\hline Secondary branches/plant & $15.07+$ & 11.46 & $18.29^{\star}$ & 12.19 \\
\hline Main shoot length (cm) & $75.86+$ & 75.76 & $80.28^{\star}$ & 78.46 \\
\hline $\begin{array}{l}\text { Point to first siliqua on main shoot } \\
(\mathrm{cm})\end{array}$ & $8.56+$ & 7.51 & $8.34^{*}$ & 7.35 \\
\hline Number of siliqua on main shoot & 48.65 & $49.97+$ & 54.30 & $55.54^{*}$ \\
\hline Total siliqua/plant & $457.48+$ & 456.80 & $539.24^{*}$ & 472.25 \\
\hline Siliqua length (cm) & 4.03 & $4.19+$ & 4.17 & $4.35^{\star}$ \\
\hline Seeds/siliqua & 15.12 & $15.98+$ & 16.00 & $16.08^{*}$ \\
\hline Days to $50 \%$ flowering & $52.67+$ & 53.96 & $57.31^{\star}$ & 55.85 \\
\hline 1,000-seed weight (g) & 3.70 & $4.49+$ & 4.00 & $4.38^{\star}$ \\
\hline Seed yield/plant (g) & 20.36 & $25.79+$ & 30.17 & $32.14^{*}$ \\
\hline Biological yield/plant (g) & 118.12 & $144.27+$ & 156.56 & $166.08^{*}$ \\
\hline Harvest index & 17.38 & $18.14+$ & 19.23 & $19.77^{*}$ \\
\hline Seed yield (kg/ha) & $2338+$ & 2300 & 2718 & $2894^{\star}$ \\
\hline Oil content (\%) & 36.63 & $38.75+$ & 38.56 & $39.07^{*}$ \\
\hline
\end{tabular}

*Traits for which higher mean value observed in hybrids.

+ Traits for which higher mean value observed in parental CDLs.

$R F$, rainfed; $I R$, irrigated.

ability and other analyses. Under both water regimes, the mean squares of parents vs. hybrids were found significant for several traits, revealed good scope for the manifestation of heterosis in hybrids generated from B. carinata-derived $B$. juncea lines. Further, under both irrigated and rainfed (Tables 1, 2) conditions, the mean sum of squares due to hybrids was significant for all the traits, indicating the importance of both additive and non-additive variance. Earlier studies have also reported similar trends for additive and non-additive variance for the traits under study (Rao and Gulati, 2001; Yadav et al., 2005; Singh et al., 2006, 2013; Singh N. et al., 2015; Verma et al., 2011). Polygenic inheritance of drought tolerance is reported in many crop species including mustard (Fletcher et al., 2015). The quantitative traits are complex to understand hence methods like quantitative trait loci (QTL) mapping, marker-assisted breeding, and introgression from wild or related species are being used to improve drought tolerance (Mwadzingeni et al., 2016).

\section{Combining Ability and Gene Action}

Combining ability analysis partitions the genotypic variability into GCA $\left(\sigma^{2}\right.$ gca $)$ and SCA $\left(\sigma^{2}\right.$ sca $)$ variances representing additive and dominance effects, respectively. The higher value of $\sigma^{2}$ sca than $\sigma^{2}$ gca, along with an average degree of dominance more than unity observed for almost all the traits studied under irrigated and rainfed conditions, demonstrates the predominance of non-additive gene effects in the manifestation of heterosis (Table 3). For siliqua length and seeds per siliqua evaluated under both irrigated and rainfed condition, $\sigma^{2}$ gca were higher than $\sigma^{2}$ sca and average degree of dominance were less than unity demonstrating the role of additive gene effects in governing them. Similar results were also reported by Yadava et al. (2012) and Singh N. et al. (2015) in Indian mustard. Since additive and nonadditive gene action were governing the yield contributing traits under irrigated and rainfed conditions, multiple or reciprocal recurrent selection could be useful for the improvement of these traits. The narrow-sense heritability $\left(h^{2}\right)$ found low to moderate for almost all the traits under both the water regimes, suggesting the importance of interaction effects (Sayar et al., 2007). Since the expected genetic gain per cycle of selection will be less important, the development of hybrids involving CDLs carrying $B$. carinata genomic segments holds promise for exploitation of heterosis under rainfed conditions.

Significant GCA effects were observed for CDL161, CDL141, CDL112, CDL186, CDL101, CDL25, and CDL89 for seed yield $(\mathrm{kg} / \mathrm{ha})$ along with some of its contributing traits under rainfed condition, whereas under irrigated condition, CDL161, CDL112, CDL186, CDL101, CDL25, CDL89, and CDL106 expressed good general combining ability for seed yield ( $\mathrm{kg} / \mathrm{ha}$ ) and its contributing traits (Table 4). The results suggested the importance of additive or additive $\times$ additive gene effects which are governing these traits. Similar results have also been reported earlier using a different set of Indian mustard genotypes (Mahak et al., 2008; Singh et al., 2010, 2013; Yadava et al., 2012; Singh N. et al., 2015). Furthermore, the good general combiners for seed yield traits were not showing significant GCA effects for some of its contributing traits, hence, there is a scope of improving the combining ability among parents for yield contributing traits under both irrigated and rainfed conditions. The findings of the present study suggest that CDLs, viz., CDL161, CDL112, CDL186, CDL101, CDL25, and CDL89 possessing variable drought tolerance and expressing high GCA for seed yield along with other component traits under both rainfed and irrigated conditions can be incorporated in the breeding programme for accumulating desirable alleles for developing improved mustard cultivars suitable to water-scarce regions.

In the present study, significant SCA effects and high heterobeltiosis (>15\%) were exhibited by 36 and 30 hybrids under rainfed and irrigated condition, respectively. Out of these, nine and six hybrids were expressing more than 50\% heterobeltiosis when evaluated under rainfed and irrigated conditions, respectively (Table 5). This suggests that CDLs taken in this study can be exploited for developing hybrids for water-scarce regions. It is also important to note that 14 hybrids exhibited higher SCA effects and heterobeltiosis (>15\%) under both water regimes (Table 6). These hybrids, expected to carry different introgression segments from $B$. carinata, have better resilience to changing water regimes and, thus, expressed better stability of performance. Earlier studies have recorded similar observations with a different set of conventional B. juncea genotypes (Singh et al., 2006; Srivastava et al., 2009). Teklewold and Becker (2005) reported significant SCA effects along with high heterobeltiosis for seed yield in B. carinata inbred lines, indicating substantial potential of Ethiopian mustard genotypes and their significance in the exploitation of hybrid vigour in $B$. juncea breeding programmes. Considerable heterosis was also reported in inter-subgenomic hybrids between 
B. carinata-derived introgression lines and $B$. juncea accessions (Wei et al., 2016). Therefore, the development of hybrids using $B$. carnata derived lines will be an ideal approach in realizing higher yields under drought conditions.

The higher SCA effects and heterobeltiosis (>15\%) for seed yield were exhibited by hybrids derived from CDLs, irrespective of their GCA effect values. Thus, hybrids generated from CDLs with significant $\times$ significant, significant $\times$ non-significant, and non-significant $\times$ non-significant GCA effects expressed high SCA effects and heterobeltiosis (Table 6). It demonstrates that joint effects of native and alien heterotic loci are responsible for the expression of such SCA effects and heterobeltiosis in hybrids derived from CDLs. Langham (1961) and Lal et al. (2006) have explained this anomaly where parents with other than high $\times$ high GCA effects also resulted in better heterosis and concluded that cross between parents with significantly high $\times$ low trait value resulted in superior transgressive segregants and, thus, the genes governing high trait values may express under homozygous or heterozygous conditions. Further, the genes governing lower trait values can also be expressed to their full potential in the homozygous state, but in heterozygote state, they can express very high performance due to complementation of alleles or overdominance. The existence of heterotic loci in these $B$. carinata derived lines can thus be exploited in the $B$. juncea breeding program for improving the level of heterosis and its commercial use.

\section{Mean Performance, Tolerance Indices, and Water Use Efficiency}

Brassica carinata, in general, adapted to tolerate drought conditions (Thakur et al., 2021), performed better under less availability of water and lodges under irrigated conditions due to its poor ability to withstand accumulated higher biomass. It has

TABLE 8 | Water use efficiency, drought tolerance indices, and seed yield performance of CDLs and their $F_{1}$ hybrids under rainfed (RF) and irrigated (IR) conditions.

\begin{tabular}{|c|c|c|c|c|c|c|c|c|c|c|c|c|c|}
\hline \multirow[t]{2}{*}{ CDLs/Cross } & \multicolumn{2}{|c|}{ Seed yield (kg/ha) } & \multicolumn{2}{|c|}{ WUE $\left(\mathrm{kg} / \mathrm{m}^{3}\right)$} & \multirow[t]{2}{*}{ DTI } & \multirow[t]{2}{*}{ MRP } & \multirow[t]{2}{*}{ CDLs/Cross } & \multicolumn{2}{|c|}{ Seed yield (kg/ha) } & \multicolumn{2}{|c|}{ WUE $\left(\mathrm{kg} / \mathrm{m}^{3}\right)$} & \multirow[t]{2}{*}{ DTI } & \multirow[t]{2}{*}{ MRP } \\
\hline & $\mathbf{R F}$ & IR & RF & IR & & & & RF & IR & $\mathbf{R F}$ & IR & & \\
\hline CDL102 & 2388 & 2281 & 2.12 & 1.07 & 0.76 & 1.70 & CDL161 × CDL186 & 3711 & 3280 & 3.30 & 1.54 & 1.71 & 2.54 \\
\hline CDL161 & 2656 & 1791 & 2.36 & 0.84 & 0.67 & 1.61 & CDL161 × CDL182 & 2983 & 2280 & 2.65 & 1.07 & 0.95 & 1.91 \\
\hline CDL128 & 2914 & 2300 & 2.59 & 1.08 & 0.94 & 1.89 & CDL161 × CDL121 & 2894 & 2635 & 2.58 & 1.24 & 1.07 & 2.01 \\
\hline CDL141 & 2478 & 1724 & 2.20 & 0.81 & 0.60 & 1.52 & CDL161 × CDL103 & 3000 & 2346 & 2.67 & 1.10 & 0.99 & 1.94 \\
\hline CDL112 & 2842 & 2713 & 2.53 & 1.28 & 1.08 & 2.02 & CDL161 × CDL101 & 3944 & 3460 & 3.51 & 1.63 & 1.91 & 2.69 \\
\hline CDL186 & 2294 & 2138 & 2.04 & 1.01 & 0.69 & 1.61 & CDL161 × CDL25 & 2567 & 3224 & 2.28 & 1.52 & 1.16 & 2.12 \\
\hline CDL182 & 2542 & 1741 & 2.26 & 0.82 & 0.62 & 1.55 & CDL161 × CDL89 & 3446 & 2791 & 3.07 & 1.31 & 1.35 & 2.27 \\
\hline CDL121 & 1506 & 2488 & 1.34 & 1.17 & 0.53 & 1.47 & CDL161 × CDL104 & 2647 & 2335 & 2.35 & 1.10 & 0.87 & 1.81 \\
\hline CDL103 & 1425 & 1559 & 1.27 & 0.73 & 0.31 & 1.09 & CDL161 × CDL105 & 3267 & 2836 & 2.91 & 1.34 & 1.30 & 2.22 \\
\hline CDL101 & 2817 & 2763 & 2.51 & 1.30 & 1.09 & 2.03 & CDL161 × CDL106 & 3046 & 2577 & 2.71 & 1.21 & 1.10 & 2.05 \\
\hline CDL25 & 2465 & 2799 & 2.19 & 1.32 & 0.97 & 1.92 & CDL128 × CDL141 & 3371 & 3018 & 3.00 & 1.42 & 1.43 & 2.33 \\
\hline CDL89 & 2511 & 3194 & 2.23 & 1.50 & 1.12 & 2.09 & CDL128 × CDL112 & 1783 & 2099 & 1.59 & 0.99 & 0.52 & 1.42 \\
\hline CDL104 & 1972 & 2630 & 1.75 & 1.24 & 0.73 & 1.68 & CDL128 × CDL186 & 3500 & 2835 & 3.11 & 1.33 & 1.39 & 2.30 \\
\hline CDL105 & 2017 & 2927 & 1.79 & 1.38 & 0.83 & 1.81 & CDL128 × CDL182 & 3864 & 2913 & 3.44 & 1.37 & 1.58 & 2.46 \\
\hline CDL106 & 1668 & 2030 & 1.48 & 0.96 & 0.47 & 1.35 & CDL128 $\times$ CDL121 & 1984 & 2283 & 1.76 & 1.07 & 0.63 & 1.56 \\
\hline CDL102 × CDL161 & 2567 & 3307 & 2.28 & 1.56 & 1.19 & 2.15 & CDL128 × CDL103 & 2835 & 2019 & 2.52 & 0.95 & 0.80 & 1.76 \\
\hline CDL102 × CDL128 & 2106 & 2696 & 1.87 & 1.27 & 0.80 & 1.76 & CDL128 × CDL101 & 3114 & 2613 & 2.77 & 1.23 & 1.14 & 2.08 \\
\hline CDL102 $\times$ CDL141 & 2372 & 2292 & 2.11 & 1.08 & 0.76 & 1.70 & CDL128 × CDL25 & 3264 & 2639 & 2.90 & 1.24 & 1.21 & 2.15 \\
\hline CDL102 × CDL112 & 2444 & 3280 & 2.17 & 1.54 & 1.12 & 2.10 & CDL128 × CDL89 & 2832 & 2087 & 2.52 & 0.98 & 0.83 & 1.79 \\
\hline CDL102 × CDL186 & 3411 & 3004 & 3.03 & 1.41 & 1.44 & 2.33 & CDL128 × CDL104 & 3233 & 2599 & 2.88 & 1.22 & 1.18 & 2.12 \\
\hline CDL102 × CDL182 & 1603 & 1694 & 1.43 & 0.80 & 0.38 & 1.20 & CDL128 × CDL105 & 2069 & 2294 & 1.84 & 1.08 & 0.67 & 1.59 \\
\hline CDL102 × CDL121 & 1411 & 1263 & 1.26 & 0.59 & 0.25 & 0.97 & CDL128 × CDL106 & 2117 & 2294 & 1.88 & 1.08 & 0.68 & 1.61 \\
\hline CDL102 × CDL103 & 2178 & 2080 & 1.94 & 0.98 & 0.64 & 1.55 & CDL141 x CDL112 & 3403 & 2689 & 3.03 & 1.27 & 1.28 & 2.21 \\
\hline CDL102 × CDL101 & 3389 & 2794 & 3.02 & 1.32 & 1.33 & 2.25 & CDL141 × CDL186 & 3094 & 2506 & 2.75 & 1.18 & 1.09 & 2.04 \\
\hline CDL102 × CDL25 & 3600 & 3857 & 3.20 & 1.82 & 1.95 & 2.72 & CDL141 × CDL182 & 3308 & 2713 & 2.94 & 1.28 & 1.26 & 2.19 \\
\hline CDL102 × CDL89 & 3807 & 3324 & 3.39 & 1.57 & 1.77 & 2.60 & CDL141 × CDL121 & 3000 & 2182 & 2.67 & 1.03 & 0.92 & 1.88 \\
\hline CDL102 × CDL104 & 2022 & 1811 & 1.80 & 0.85 & 0.51 & 1.40 & CDL141 × CDL103 & 2790 & 2335 & 2.48 & 1.10 & 0.91 & 1.86 \\
\hline CDL102 × CDL105 & 1922 & 1602 & 1.71 & 0.75 & 0.43 & 1.28 & CDL141 × CDL101 & 2572 & 2785 & 2.29 & 1.31 & 1.00 & 1.96 \\
\hline CDL102 × CDL106 & 3722 & 3113 & 3.31 & 1.47 & 1.62 & 2.49 & CDL141 × CDL25 & 3475 & 2816 & 3.09 & 1.33 & 1.37 & 2.29 \\
\hline CDL161 × CDL128 & 3906 & 3313 & 3.47 & 1.56 & 1.81 & 2.63 & CDL141 × CDL89 & 2700 & 1819 & 2.40 & 0.86 & 0.69 & 1.64 \\
\hline CDL161 × CDL141 & 3800 & 3265 & 3.38 & 1.54 & 1.74 & 2.57 & CDL141 x CDL104 & 2800 & 2037 & 2.49 & 0.96 & 0.80 & 1.76 \\
\hline CDL161 × CDL112 & 2561 & 2730 & 2.28 & 1.29 & 0.98 & 1.93 & CDL141 × CDL105 & 2304 & 2196 & 2.05 & 1.03 & 0.71 & 1.64 \\
\hline
\end{tabular}


TABLE 8 | (Continued)

\begin{tabular}{|c|c|c|c|c|c|c|c|c|c|c|c|c|c|}
\hline \multirow[t]{2}{*}{ CDLs/Cross } & \multicolumn{2}{|c|}{ Seed yield (kg/ha) } & \multicolumn{2}{|c|}{ WUE $\left(\mathbf{k g} / \mathrm{m}^{3}\right)$} & \multirow[t]{2}{*}{ DTI } & \multirow[t]{2}{*}{ MRP } & \multirow[t]{2}{*}{ CDLs/Cross } & \multicolumn{2}{|c|}{ Seed yield (kg/ha) } & \multicolumn{2}{|c|}{ WUE $\left(\mathrm{kg} / \mathrm{m}^{3}\right)$} & \multirow[t]{2}{*}{ DTI } & \multirow[t]{2}{*}{ MRP } \\
\hline & RF & IR & $\mathbf{R F}$ & IR & & & & RF & IR & RF & IR & & \\
\hline CDL141 × CDL106 & 2828 & 2107 & 2.52 & 0.99 & 0.84 & 1.79 & CDL121 × CDL103 & 2503 & 3021 & 2.23 & 1.42 & 1.06 & 2.02 \\
\hline CDL112 × CDL186 & 2892 & 2291 & 2.57 & 1.08 & 0.93 & 1.88 & CDL121 × CDL101 & 2022 & 2602 & 1.80 & 1.22 & 0.74 & 1.69 \\
\hline CDL112 × CDL182 & 3050 & 2996 & 2.71 & 1.41 & 1.28 & 2.20 & CDL121 × CDL25 & 3597 & 3119 & 3.20 & 1.47 & 1.57 & 2.44 \\
\hline CDL112 × CDL121 & 2839 & 3335 & 2.53 & 1.57 & 1.33 & 2.26 & CDL121 × CDL89 & 2658 & 2482 & 2.37 & 1.17 & 0.93 & 1.87 \\
\hline CDL112 × CDL103 & 2783 & 2313 & 2.48 & 1.09 & 0.90 & 1.85 & CDL121 × CDL104 & 2597 & 2857 & 2.31 & 1.35 & 1.04 & 1.99 \\
\hline CDL112 × CDL101 & 2558 & 2924 & 2.28 & 1.38 & 1.05 & 2.00 & CDL121 × CDL105 & 2469 & 2280 & 2.20 & 1.07 & 0.79 & 1.73 \\
\hline CDL112 $\times$ CDL25 & 3052 & 2993 & 2.72 & 1.41 & 1.28 & 2.20 & CDL121 × CDL106 & 2829 & 2202 & 2.52 & 1.04 & 0.87 & 1.83 \\
\hline CDL112 × CDL89 & 4333 & 4191 & 3.86 & 1.97 & 2.55 & 3.11 & CDL103 × CDL101 & 1857 & 3263 & 1.65 & 1.54 & 0.85 & 1.88 \\
\hline CDL112 × CDL104 & 3083 & 2291 & 2.74 & 1.08 & 0.99 & 1.95 & CDL103 × CDL25 & 2795 & 3113 & 2.49 & 1.47 & 1.22 & 2.16 \\
\hline CDL112 × CDL105 & 2725 & 3002 & 2.42 & 1.41 & 1.15 & 2.09 & CDL103 × CDL89 & 1801 & 2757 & 1.60 & 1.30 & 0.70 & 1.67 \\
\hline CDL112 × CDL106 & 3333 & 2968 & 2.97 & 1.40 & 1.39 & 2.29 & CDL103 × CDL104 & 1142 & 1957 & 1.02 & 0.92 & 0.31 & 1.14 \\
\hline CDL186 × CDL182 & 1869 & 2313 & 1.66 & 1.09 & 0.61 & 1.53 & CDL103 × CDL105 & 2756 & 2060 & 2.45 & 0.97 & 0.80 & 1.75 \\
\hline CDL186 × CDL121 & 3836 & 3718 & 3.41 & 1.75 & 2.00 & 2.75 & CDL103 × CDL106 & 2469 & 2555 & 2.20 & 1.20 & 0.88 & 1.83 \\
\hline CDL186 × CDL103 & 2042 & 1768 & 1.82 & 0.83 & 0.51 & 1.39 & CDL101 × CDL25 & 3036 & 3863 & 2.70 & 1.82 & 1.64 & 2.52 \\
\hline CDL186 × CDL101 & 3447 & 3946 & 3.07 & 1.86 & 1.91 & 2.70 & CDL101 × CDL89 & 3168 & 2430 & 2.82 & 1.14 & 1.08 & 2.03 \\
\hline CDL186 × CDL25 & 3186 & 2604 & 2.83 & 1.23 & 1.16 & 2.11 & CDL101 × CDL104 & 4008 & 3252 & 3.57 & 1.53 & 1.83 & 2.64 \\
\hline CDL186 × CDL89 & 3108 & 3468 & 2.76 & 1.63 & 1.51 & 2.40 & CDL101 × CDL105 & 2400 & 2116 & 2.14 & 1.00 & 0.71 & 1.64 \\
\hline CDL186 × CDL104 & 3419 & 2557 & 3.04 & 1.20 & 1.23 & 2.17 & CDL101 × CDL106 & 3608 & 3932 & 3.21 & 1.85 & 1.99 & 2.75 \\
\hline CDL186 × CDL105 & 2936 & 2343 & 2.61 & 1.10 & 0.96 & 1.92 & CDL25 $\times$ CDL89 & 3212 & 3067 & 2.86 & 1.44 & 1.38 & 2.29 \\
\hline CDL186 × CDL106 & 3612 & 2752 & 3.21 & 1.30 & 1.39 & 2.31 & CDL25 × CDL104 & 4217 & 3263 & 3.75 & 1.54 & 1.93 & 2.72 \\
\hline CDL182 × CDL121 & 4296 & 3694 & 3.82 & 1.74 & 2.23 & 2.91 & CDL25 × CDL105 & 2422 & 2610 & 2.16 & 1.23 & 0.89 & 1.84 \\
\hline CDL182 × CDL103 & 2386 & 2802 & 2.12 & 1.32 & 0.94 & 1.90 & CDL25 × CDL106 & 3142 & 2902 & 2.80 & 1.37 & 1.28 & 2.20 \\
\hline CDL182 × CDL101 & 3156 & 2885 & 2.81 & 1.36 & 1.28 & 2.20 & CDL89 × CDL104 & 3972 & 3368 & 3.53 & 1.59 & 1.88 & 2.67 \\
\hline CDL182 × CDL25 & 3022 & 2374 & 2.69 & 1.12 & 1.01 & 1.96 & CDL89 × CDL105 & 2092 & 2327 & 1.86 & 1.10 & 0.68 & 1.61 \\
\hline CDL182 × CDL89 & 3381 & 2801 & 3.01 & 1.32 & 1.33 & 2.25 & CDL89 × CDL106 & 3342 & 2921 & 2.97 & 1.38 & 1.37 & 2.28 \\
\hline CDL182 × CDL104 & 2278 & 3352 & 2.03 & 1.58 & 1.07 & 2.06 & CDL104 × CDL105 & 2500 & 1880 & 2.22 & 0.88 & 0.66 & 1.59 \\
\hline CDL182 × CDL105 & 1753 & 2055 & 1.56 & 0.97 & 0.50 & 1.39 & CDL104 × CDL106 & 3936 & 4520 & 3.50 & 2.13 & 2.49 & 3.09 \\
\hline \multirow[t]{3}{*}{ CDL182 × CDL106 } & 2667 & 3238 & 2.37 & 1.52 & 1.21 & 2.16 & CDL105 × CDL106 & 1669 & 1624 & 1.48 & 0.76 & 0.38 & 1.20 \\
\hline & & & & & & & Overall mean & 2819 & 2670 & 2.51 & 1.26 & 1.09 & 2.00 \\
\hline & & & & & & & $\mathrm{CD}$ & 321 & 318 & & & & \\
\hline
\end{tabular}

WUE, water use efficiency; DTI, drought tolerance index; MRP, mean relative performance.

also been observed that irrigations in B. carinata genotypes also exhibits heavy regeneration, resulting in reduced plant height, poor seed set, and reduced seed size, which ultimately leads to reduction in seed yield per unit area. Conversely, bolder seed size is realised under rainfed than in irrigated conditions.

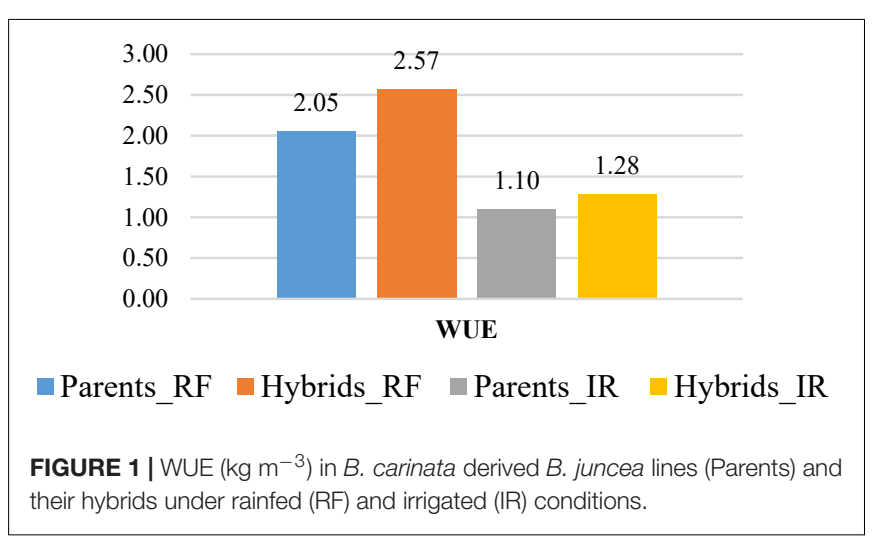

In the present study, some of the B. carinata derived lines, along with their hybrids, observed reduced performance for seed yield under irrigated than in rainfed conditions. This could be attributed to the introduction of genomic segment(s) or substituted chromosomes from the $\mathrm{C}$ genome of $B$. carinata to $B$. juncea. Although, the possibility of later is remote when the biparental mating approach is being used for the development of such CDLs.

Parental CDLs and their hybrids exhibited higher mean values for seed yield $(\mathrm{kg} / \mathrm{ha})$ and other yield attributes under rainfed than in irrigated condition (Table 7). It is most probably due to presence of different introgression segments in CDLs which alter their performance in water deficit and surplus condition, whereas complementation of distinct genomic segments and expressed overdominance leads to heterosis in hybrids. Therefore, comparatively better performance of these hybrids under rainfed compared to irrigated conditions suggested that $B$. carinata-derived lines hold promise for the development of hybrids or cultivars for water-scarce regions. 

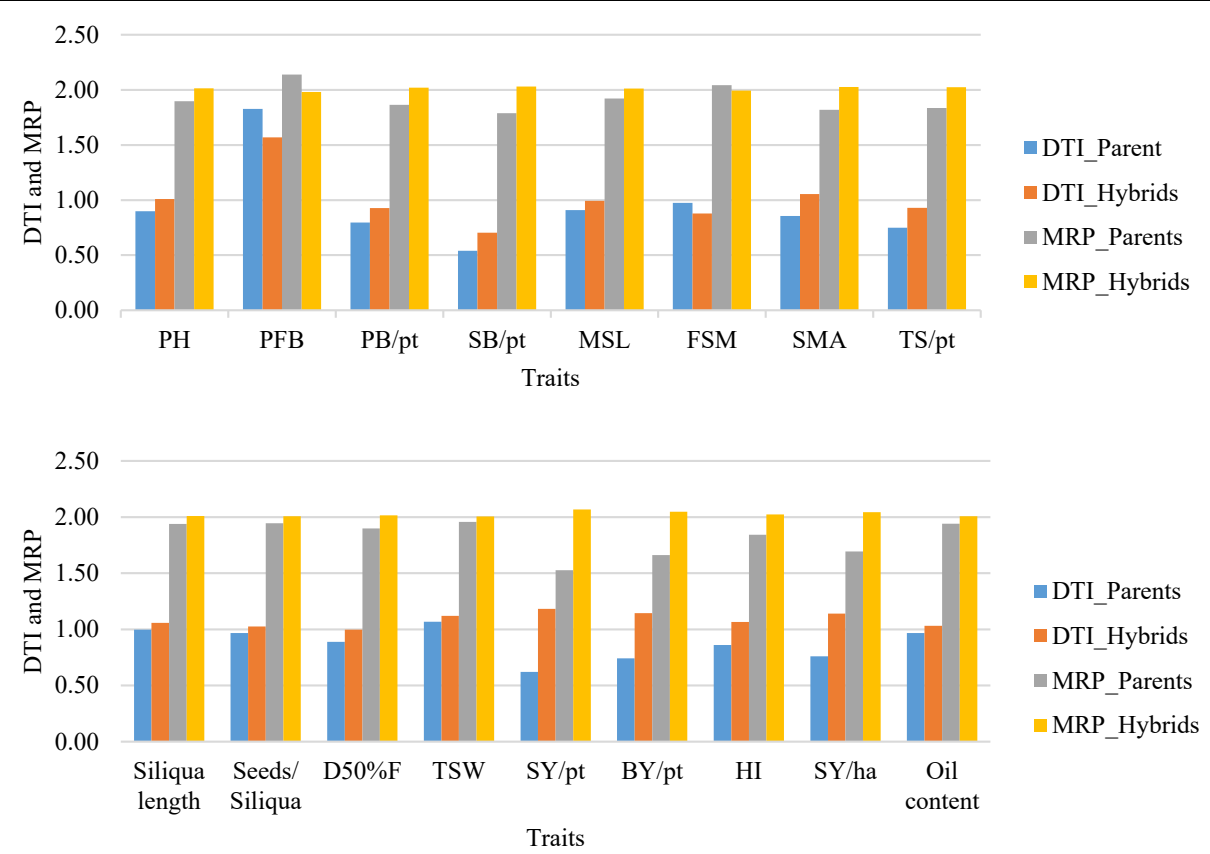

FIGURE 2 | The drought tolerant index (DTI) and mean relative performance (MRP) observed on seventeen traits in hybrids along with parents grown in rainfed and irrigated conditions. PH, plant height; PFB, point to first branch; PB/pt, primary branches/plant, SB/pt, secondary branches/plant; MSL, main shoot length; FSM, point to first siliqua on main shoot; SMS, number of siliquae on main shoot; TS/pt, total siliquae/plant; D50\%F, day to 50\% flowering; TSW, thousand seed weight; $\mathrm{SY} / \mathrm{pt}$, seed yield/plant; BY/pt, biological yield/plant; HI, harvest index; SY/ha, seed yield kg per hectare.

The present study revealed that yield under rainfed condition $\left(\mathrm{Y}_{R F}\right)$ was showing positive correlation with $\mathrm{WUE}_{R F}(r=0.99)$, WUE $_{I R}(r=0.65)$, and stress tolerance indices, viz., DTI $(r=0.90)$, MRP $(r=0.92)$, and TOL $(r=0.54)$. Similarly, yield under irrigated conditions $\left(\mathrm{Y}_{I R}\right)$ was positively correlated with DTI $(r=0.89)$ and MRP $(r=0.90)$. In addition, DTI was found positively correlated with MRP $(r=0.99)$ (Supplementary Table 2). In the present study, a higher mean seed yield was recorded in rainfed than in irrigated conditions. Therefore, DSI would not be a reliable criterion for selection when mean values under stress condition is higher than that of normal irrigated condition. Better performance of CDLs and their hybrids under rainfed condition compared to irrigated plots attributed to excessive lodging of plants in irrigated conditions. Although the level of this lodging in CDLs and hybrids is much less than what normally happens in B. carinata after irrigation or rainfall. Therefore, DTI and MRP were found to be better predictors of $\mathrm{Y}_{I R}$ and $\mathrm{Y}_{R F}$ than TOL and DSI. This study is in accordance with previous reports suggesting DTI as a better parameter for identifying drought-tolerant genotypes in mungbean (Fernandez, 1992), corn (Shirinzadeh et al., 2010), wheat (Farshadfar et al., 2012), chickpea (Pour-Siahbidi and Pour-Aboughadareh, 2013), durum wheat (Mohammadi, 2016), and barley (Khalili et al., 2016). Mahajan et al. (2018) also reported that DTI is a more desirable index for stress tolerance than SSI in rice under moisture deficit stress.

The test hybrids expressed improved WUE as compared to parental CDLs. Overall, the mean of seed yield ( $\mathrm{kg} / \mathrm{ha})$ and WUE of parental CDLs and hybrids was recorded higher under rainfed condition than in irrigated conditions (Figure 1), indicating the presence of putatively different loci/genomic segments in the CDLs for improved WUE and yield heterosis. Expression of overdominance and/or complementation of favourable alleles in hybrids appear to be the reason for improved WUE and established genetic elite in the hybrids.

Higher MRP and DTI confirmed better tolerance/adaptation of hybrids to moisture deficit stress over the parental CDLs for most of the traits under study (Figure 2). Hybrids expressing DTI value of $>1$, indicating their superiority under both moisture-stress and non-stress conditions. Lower DTI value, on the other hand, did not precisely explain susceptibility to moisture deficit stress conditions as mean yield under rainfed was higher than irrigated in present study. CDLs carry introgressions from $B$. carinata lodged after irrigation or rain and, thus, lead to a reduction in seed yield. In general, higher MRP of hybrids than CDLs indicates better performance of hybrids under rainfed and irrigated conditions. Based on higher DTI and MRP, highly productive and drought-tolerant hybrids, viz., CDL102 × CDL186, CDL102 × CDL25, CDL102 × CDL89, CDL102 × CDL106, CDL161 × CDL128, CDL161 × CDL141, CDL161 × CDL186, CDL161 × CDL101, CDL128 × CDL141, CDL186 × CDL121, CDL186 × CDL101, CDL182 × CDL121, CDL121 × CDL25, CDL101 × CDL104, CDL101 × CDL106, CDL25 $\times$ CDL89, CDL25 $\times$ CDL104, CDL89 $\times$ CDL104, and CDL104 $\times$ CDL106 were identified (Table 8). These hybrids can be commercially exploited by converting the parental CDLs into CMS or restorer lines based on their seed production suitability. Hence, hybrid vigour expressed under moisture deficit stress 
conditions is well demonstrated through the deployment of $B$. carinata-derived $B$. juncea lines.

\section{CONCLUSION}

In the present study, the availability of larger SCA variance than GCA variance for seed yield and its contributing traits under both water deficit and water sufficient conditions indicated a preponderance of dominance gene action. It suggests the manifestation of heterosis in B. carinata-derived B. juncea lines under moisture deficit stress conditions. The predominance of additive gene effects for traits like siliqua length and seeds per siliqua suggested the involvement of genetic and $\mathrm{G} \times \mathrm{E}$ interactions for these traits. Hybrids developed by involving CDLs with desirable SCA effects, higher heterobeltiosis, and better WUE exhibit overdominance and/or complement favourable alleles among different CDLs for expression of exploitable heterosis in hybrids. Many of the B. carinata-derived $B$. juncea lines that were identified in this study were heterotic and can be exploited for the development of commercial hybrids by converting them to cytoplasmic male sterile and/or restorer lines. CDLs, on the other hand, can also be used to recover desirable recombinants that would help in hastening the Brassica improvement programmes for the drought-prone rainfed areas. Material and information emanating from this study shall open new vistas for research involving related species for interspecific hybridization among Brassica species in creating novel genetic variability and deploying them for the development of high yielding hybrids with better WUE and, thus, overcoming the yield barriers in drought-prone regions.

\section{DATA AVAILABILITY STATEMENT}

The original contributions presented in the study are included in the article/Supplementary Material, further inquiries can be directed to the corresponding author/s.

\section{REFERENCES}

Anonymous (2021). Global Agricultural Information Network Circular Series, WAP 9-21. Washington, DC: USDA Foreign Agricultural Service.

Chauhan, J. S., Singh, K. H., Singh, V. V., and Kumar, S. (2011). Hundred years of rapeseed-mustard breeding in India: accomplishments and future strategies. Indian J. Agric. Sci. 81, 1093-1109.

Choudhary, B. R., Joshi, P., and Ramarao, S. (2008). Interspecific hybridization between Brassica carinata and Brassica rapa. Plant Breed. 119, 417-420. doi: 10.1046/j.1439-0523.2000.00503.x

Clarke, J. M., Townley-Smith, F., McCaig, T. N., and Green, D. G. (1984). Growth analysis of spring wheat cultivars of varying drought resistance 1. Crop Sci. 24, 537-541. doi: 10.2135/cropsci1984.0011183X002400030026x

Coffman, F. A. (1933). Heterosis: Specific not general in nature. Science 77, 114-115. doi: 10.1126/science.77.1987.114-c

Comstock, R. E., and Robinson, H. F. (1948). The components of genetic variance in populations of biparental progenies and their use in estimating the average degree of dominance. Biometrics 4, 254-266. doi: 10.2307/3001412

Dalal, M., Tayal, D., Chinnusamy, V., and Bansal, K. C. (2009). Abiotic stress and ABA-inducible Group 4 LEA from Brassica napus plays a key role in salt

\section{AUTHOR CONTRIBUTIONS}

OL conducted the field trials, generated crosses, analysed the data, and prepared the draft manuscript. RS conducted the trial, recorded observations, and maintained the CDLs. PV helped in data recording and analysis. PK and CP helped in conducting field trials, recording observations, and data analysis. JN helped in generating test hybrids. DY supervised the research. VC guided the research and edited the manuscript. NS developed the CDLs, conceptualized and supervised the research, analysed the data, and wrote the manuscript. All the authors read the manuscript and agreed with its content.

\section{FUNDING}

The research was funded by ICAR-IARI in-house project.

\section{ACKNOWLEDGMENTS}

OL is grateful to the University Grants Commission of India and Post-graduate School, ICAR-Indian Agricultural Research Institute (IARI), New Delhi for granting Senior Research Fellowship. The authors are thankful to the Division of Genetics, ICAR-IARI, New Delhi for providing facilities for hybridisation and evaluation of CDLs and hybrids. The authors are also thankful to the ICAR-IARI, Regional Station, Wellington, Tamil Nadu for extending help and resources for generating hybrid seed in the off-season.

\section{SUPPLEMENTARY MATERIAL}

The Supplementary Material for this article can be found online at: https://www.frontiersin.org/articles/10.3389/fpls.2021. 765645/full\#supplementary-material

and drought tolerance. J. Biotechnol. 139, 137-145. doi: 10.1016/j.jbiotec.2008. 09.014

Farshadfar, E., Poursiahbidi, M. M., and Abooghadareh, A. P. (2012). Repeatability of drought tolerance indices in bread wheat genotypes. Int. J. Agric. Crop Sci. 4, 891-903. doi: 10.1016/j.jplph.2014.04.013

Fernandez, G. C. (1992). "Effective selection criteria for assessing plant stress tolerance," in Proceeding of the International Symposium on Adaptation of Vegetables and other Food Crops in Temperature and Water Stress, Shanhua, 257-270.

Fischer, R. A., and Maurer, R. (1978). Drought resistance in spring wheat cultivars. I. Grain yield responses. Austral. J. Agric. Res. 29, 897-912. doi: 10.1371/journal. pone.0199121

Fletcher, R. S., Mullen, J. L., Heiliger, A., and McKay, J. K. (2015). QTL analysis of root morphology, flowering time, and yield reveals trade-offs in response to drought in Brassica napus. J. Exp. Bot. 66, 245-256. doi: 10.1093/jxb/ eru423

Getinet, A., Rakow, G., Raney, J. P., and Downey, R. K. (1994). Development of zero erucic acid Ethiopian mustard through an interspecific cross with zero erucic acid Oriental mustard. Can. J. Plant Sci. 74, 793-795. doi: 10.4141/ cjps94- 141 
Griffing, B. R. U. C. E. (1956). Concept of general and specific combining ability in relation to diallel crossing systems. Austral. J. Biol. Sci. 9, 463-493. doi: 10.1071/BI9560463

Huang, B. (2000). Role of Root Morphological and Physiological Characteristics in Drought Resistance of Plants. Plant-Environment Interactions. New York: Marcel Dekker Inc, 39-64. doi: 10.1201/9780824746568.ch2

Inomata, N. (1997). "Wide hybridization and meiotic pairing," in Recent Advances in Oilseed Brassicas, eds H. R. Kalia and S. K. Gupta (Ludhiana: Kalyani Publ.), 53-76.

Jiang, Y., Tian, E., Li, R., Chen, L., and Meng, J. (2007). Genetic diversity of Brassica carinata with emphasis on the interspecific crossability with $B$. rapa. Plant Breed. 126, 487-491. doi: 10.1111/j.1439-0523.2007.01393.x

Kasuga, M., Liu, Q., Miura, S., Yamaguchi-Shinozaki, K., and Shinozaki, K. (1999). Improving plant drought, salt, and freezing tolerance by gene transfer of a single stress-inducible transcription factor. Nat. Biotechnol. 17, 287-291. doi: 10.1038/7036

Khalili, M., Pour-Aboughadareh, A., and Naghavi, M. R. (2016). Assessment of drought tolerance in barley: integrated selection criterion and drought tolerance indices. Environ. Exp. Biol. 14, 33-41. doi: 10.22364/eeb.14.06

Khan, S. A., Ahmad, H., Khan, A., Saeed, M., Khan, S. M., and Ahmad, B. (2009). Using line $\mathrm{x}$ tester analysis for earliness and plant height traits in sunflower (Helianthus annuus L.). Recent Res. Sci. Technol. 1:316.

Lal, C., Hariprasanna, K., Rathnakumar, A. L., Gor, H. K., and Chikani, B. M. (2006). Gene action for surrogate traits of water-use efficiency and harvest index in peanut (Arachis hypogaea). Ann. Appl. Biol. 148, 165-172. doi: 10.1111/j. 1744-7348.2006.00047.x

Langham, D. G. (1961). The high-low method in crop improvement. Crop Sci. 1, 376-378. doi: 10.2135/cropsci1961.0011183X000100050026x

Mahajan, G., Singh, K., Singh, N., Kaur, R., and Chauhan, B. S. (2018). Screening of water-efficient rice genotypes for dry direct seeding in South Asia. Arch. Agron. Soil Sci. 64, 103-115. doi: 10.1080/03650340.2017.1337891

Mahak, S., Bashrat, A. M., Lokendra, S., Brahm, S., and Dixit, R. K. (2008). Studies on combining ability for oil content, seed yield and its contributing characters in Indian mustard [Brassica juncea (L.) Czern \& and Coss]. Prog. Res. 3, 147-150.

Malik, R. S. (1990). Prospects for Brassica carinata as an oilseed crop in India. Exp. Agric. 26, 125-129. doi: 10.1017/s0014479700015465

Mather, K., and Jinks, J. L. (1982). Biometrical Genetics: the Study of Continuous Variation, 3rd Edn. New York, NY: Chapman and Hall Publishers.

Mohammadi, R. (2016). Efficiency of yield-based drought tolerance indices to identify tolerant genotypes in durum wheat. Euphytica 211, 71-89.

Mwadzingeni, L., Shimelis, H., Dube, E., Laing, M. D., and Tsilo, T. J. (2016). Breeding wheat for drought tolerance: progress and technologies. J. Integr. Agric. 15, 935-943.

Navabi, Z. K., Stead, K. E., Pires, J. C., Xiong, Z., Sharpe, A. G., Parkin, I. A., et al. (2011). Analysis of B-genome chromosome introgression in interspecific hybrids of Brassica napus $\times$ B. carinata. Genetics 187, 659-673. doi: 10.1534/ genetics.110.124925

Panhwar, S. A., Baloch, M. J., Jatoi, W. A., Veesar, N. F., and Majeedano, M. S. (2008). Combining ability estimates from line $\mathrm{x}$ tester mating design in upland cotton. Proc. Pakistan Acad. Sci. 45, 69-74.

Pour-Siahbidi, M. M., and Pour-Aboughadareh, A. (2013). Evaluation of grain yield and repeatability of drought tolerance indices for screening chickpea (Cicer aritinum L.) genotypes under rainfed conditions. Iranian J. Genet. Plant Breed. $2,28-37$.

Prakash, S. (1973). Non-homologous meiotic pairing in the A and B genomes of Brassica: its breeding significance in the production of variable amphidiploids. Genet. Res. Camb. 21, 133-137. doi: 10.1017/s0016672300013318

Raman, A., Verulkar, S., Mandal, N., Variar, M., Shukla, V., Dwivedi, J., et al. (2012). Drought yield index to select high yielding rice lines under different drought stress severities. Rice 5:31. doi: 10.1186/1939-8433-5-31

Raman, R., Qiu, Y., Coombes, N., Song, J., Kilian, A., and Raman, H. (2017). Molecular diversity analysis and genetic mapping of pod shatter resistance loci in Brassica carinata L. Front. Plant Sci. 8:1765. doi: 10.3389/fpls.2017.01765

Rao, N. V. P. R. G., and Gulati, S. C. (2001). Combining ability of gene action in F1 and F2 diallels of Indian mustard. Crop Res. Hisar 21, 72-76.
Rosielle, A. A., and Hamblin, J. (1981). Theoretical aspects of selection for yield in stress and non-stress environment 1. Crop Sci. 21, 943-946. doi: 10.2135/ cropsci1981.0011183x002100060033x

Sayar, R., Khemira, H., and Kharrat, M. (2007). Inheritance of deeper root length and grain yield in half-diallel durum wheat (Triticum durum) crosses. Ann. Appl. Biol. 151, 213-220. doi: 10.1111/j.1744-7348.2007. 00168.x

Seo, Y. J., Park, J. B., Cho, Y. J., Jung, C., Seo, H. S., Park, S. K., et al. (2010). Overexpression of the ethylene-responsive factor gene BrERF4 from Brassica rapa increases tolerance to salt and drought in Arabidopsis plants. Mol. Cells 30, 271-277. doi: 10.1007/s10059-010-0114-z

Sharma, D. K., and Kumar, A. (1989). Effect of water stress on plant water relations and yield of varieties of Indian mustard (Brassica juncea). Indian J. Agric. Sci. 59, 281-285.

Sharma, P., Sharma, H. O., and Rai, P. K. (2018). Strategies and technologies for enhancing rapeseed-mustard production and farmer income. Indian Farming $68,44-48$.

Sheikh, F. A., Bangha, S., and Banga, S. S. (2014). Broadening the genetic base of Abyssinian mustard (Brassica carinata A. Braun) through introgression of genes from related allotetraploid species. Span. J. Agric. Res. 12, 742-752. doi: 10.5424/sjar/2014123-5365

Shekhawat, K., Rathore, S. S., Premi, O. P., Kandpal, B. K., and Chauhan, J. S. (2012). Advances in agronomic management of Indian mustard (Brassica juncea (L.) Czernj. Cosson): an overview. Int. J. Agron. 2012:408284. doi: $10.1155 / 2012 / 408284$

Shirinzadeh, A., Zarghami, R., Azghandi, A. V., Shiri, M. R., and Mirabdulbaghi, M. (2010). Evaluation of drought tolerance in mid and late mature corn hybrids using stress tolerance indices. Asian J. Plant Sci. 9, 67-73. doi: 10.3923/ajps. 2010.67 .73

Singh, A., Avtar, R., Singh, D., Sangwan, O., Thakral, N. K., Malik, V. S., et al. (2013). Combining ability analysis for seed yield and component traits in Indian mustard [Brassica juncea (L.) Czern \& Coss.]. Res. Plant Biol. $3,26-31$.

Singh, K. H., and Chauhan, J. S. (2010). Morphological Descriptor of Rapeseed Mustard Varieties. Sewar: Directorate of Rapeseed Mustard Research.

Singh, K. H., Shakya, R., Singh, K. K., Thakur, A. K., Nanjundan, J., and Singh, D. (2015). "Genetic enhancement of Brassica carinata through interspecific hybridization and population improvement," in Proceedings of the 14th International Rapeseed Congress, Saskatoon.

Singh, M., Satendra, S. H., and Dixit, R. K. (2006). Combining ability of agronomic characters in Indian mustard (Brassica juncea (L.) Czern \& and Coss). J. Prog. Res. 6, 69-72.

Singh, M., Singh, L., and Srivastava, S. B. L. (2010). Combining ability analysis in Indian mustard (Brassica juncea L. Czern \& Coss). J. Oilseed Brassica $1,23-27$.

Singh, N., Yadava, D. K., Sujata, V., Singh, R., Giri, S. C., Dass, B., et al. (2015). Combining ability and heterobeltiosis for yield and yield contributing traits in high quality oil Indian mustard (Brassica juncea) genotypes. Indian J. Agric. Sci. $85,498-503$.

Srivastava, S. B. L., Rajshekhar, Singh, M., and Rao, M. (2009). Combining ability analysis for yield and contributing characters in Indian mustard (Brassica juncea (L.) Czern \& and Coss.). J. Oil Seeds Res. 26, 58-61. doi: 10.9734/cjast/ 2020/v39i2030808

Teklewold, A., and Becker, H. C. (2005). Heterosis and combining ability in a diallel cross of Ethiopian mustard inbred lines. Crop Sci. 45, 2629-2635. doi: $10.2135 /$ cropsci2005.0085

Thakur, A. K., Singh, K. H., Parmar, N., Sharma, D., Mishra, D. C., Singh, L., et al. (2021). Population structure and genetic diversity as revealed by SSR markers in Ethiopian mustard (Brassica carinata A. Braun): a potential edible and industrially important oilseed crop. Genet. Resour. Crop Evol. 68, 321-333. doi: 10.1007/s10722-020-00988-3

Thakur, A. K., Singh, K. H., Sharma, D., Parmar, N., and Nanjundan, J. (2019). Breeding and genomics interventions in Ethiopian mustard (Brassica carinata A. Braun) improvement-A mini review. S. Afr. J. Bot. 125, 457-465. doi: 10.1016/j.sajb.2019.08.002 
Verma, O. P., Rashmi, Y., Kumar, K., Ranjeet, S., and Maurya, K. N. (2011). Combining ability and heterosis for seed yield and its components in Indian mustard (Brassica juncea L. Czern \&,Coss.). Plant Arch. 11, 863-865.

Warwick, S. I. (ed.) (1993). "Wild species in the tribe Brassicaceae (Cruciferae) as sources of agronomic traits," in Guide to the Wild Germplasm of Brassica and Allied Crops, (Ottawa: Center for Land and Biological Resources Research Branch, Agriculture Canada).

Wei, Z., Wang, M., Chang, S., Wu, C., Liu, P., Meng, J., et al. (2016). Introgressing subgenome components from Brassica rapa and B. carinata to B. juncea for broadening its genetic base and exploring intersubgenomic heterosis. Front. Plant Sci. 7:1677. doi: 10.3389/fpls.2016.01677

Yadav, Y. P., Prakash, R., Singh, R., Singh, R. K., and Yadava, J. S. (2005). Genetics of yield and its component characters in Indian mustard, Brassica juncea (L.) Czern \& Coss under rainfed conditions. J. Oilseeds Res. 22:255.

Yadava, D. K., Singh, N., Sujata, V., Singh, R., Singh, S., Giri, S. C., et al. (2012). Combining ability and heterobeltiosis for yield and yield attributing traits in Indian mustard (Brassica juncea). Indian J. Agric. Sci. 82, 563-570.
Conflict of Interest: The authors declare that the research was conducted in the absence of any commercial or financial relationships that could be construed as a potential conflict of interest.

Publisher's Note: All claims expressed in this article are solely those of the authors and do not necessarily represent those of their affiliated organizations, or those of the publisher, the editors and the reviewers. Any product that may be evaluated in this article, or claim that may be made by its manufacturer, is not guaranteed or endorsed by the publisher.

Copyright (C) 2021 Limbalkar, Singh, Kumar, Nanjundan, Parihar, Vasisth, Yadava, Chinnusamy and Singh. This is an open-access article distributed under the terms of the Creative Commons Attribution License (CC BY). The use, distribution or reproduction in other forums is permitted, provided the original author(s) and the copyright owner(s) are credited and that the original publication in this journal is cited, in accordance with accepted academic practice. No use, distribution or reproduction is permitted which does not comply with these terms. 